\title{
Phenotypic Analyses of Arabidopsis T-DNA Insertion Lines and Expression Profiling Reveal That Multiple L-Type Lectin Receptor Kinases Are Involved in Plant Immunity
}

\author{
Yan Wang, ${ }^{1,2}$ Klaas Bouwmeester, ${ }^{1,3}$ Patrick Beseh, ${ }^{1}$ Weixing Shan, ${ }^{2,4}$ and Francine Govers ${ }^{1}$ \\ ${ }^{1}$ Laboratory of Phytopathology, Plant Sciences Group, Wageningen University, Wageningen, The Netherlands; ${ }^{2}$ College \\ of Plant Protection, Northwest A\&F University, Yangling, Shaanxi, P. R. China; ${ }^{3}$ Plant-Microbe Interactions, Department of \\ Biology, Faculty of Science, Utrecht University, Utrecht, The Netherlands; ${ }^{4}$ State Key Laboratory of Crop Stress Biology \\ for Arid Areas, Northwest A\&F University, Yangling, Shaanxi, P. R. China
}

Submitted 29 June 2014. Accepted 21 July 2014.

\begin{abstract}
L-type lectin receptor kinases (LecRK) are membrane-spanning receptor-like kinases with putative roles in biotic and abiotic stress responses and in plant development. In Arabidopsis, 45 LecRK were identified but their functions are largely unknown. Here, a systematic functional analysis was carried out by evaluating phenotypic changes of Arabidopsis LecRK T-DNA insertion lines in plant development and upon exposure to various external stimuli. None of the LecRK T-DNA insertion lines showed clear developmental changes, either under normal conditions or upon abiotic stress treatment. However, many of the T-DNA insertion lines showed altered resistance to Phytophthora brassicae, Phytophthora capsici, Pseudomonas syringae, or Alternaria brassicicola. One mutant defective in LecRK-V.5 expression was compromised in resistance to two Phytophthora spp. but showed enhanced resistance to Pseudomonas syringae. LecRK-V.5 overexpression confirmed its dual role in resistance and susceptibility depending on the pathogen. Combined analysis of these phenotypic data and LecRK expression profiles retrieved from public datasets revealed that LecRK which are hardly induced upon infection or even suppressed are also involved in pathogen resistance. Computed coexpression analysis revealed that LecRK with similar function displayed diverse expression patterns. Because LecRK are widespread in plants, the results presented here provide invaluable information for exploring the potential of LecRK as novel sources of resistance in crops.
\end{abstract}

Plasma membrane-localized receptor-like kinases (RLK) are critical for plants to perceive and process environmental stimuli. In Arabidopsis, there are over 400 plasma membrane-localized RLK with a conserved cytosolic kinase domain and divergent extracellular domains (Shiu and Bleecker 2003). Several of these RLK have been analyzed in detail and demonstrated to play essential roles in plant development or plant immunity. For example, the RLK CLAVATA1 controls meristem cell proliferation and differentiation (Dievart et al. 2003), and RLK

Corresponding author: F. Govers; Telephone: +31 317 483138; Fax: +31 317 483412; E-mail: Francine.Govers@wur.nl

* The $e$-Xtra logo stands for "electronic extra" and indicates that four supplementary figures and two supplementary tables are published online and that Figures 1 through 4 and Table 1 appear in color online.

(C) 2014 The American Phytopathological Society such as FLS2, EFR, and CERK1 recognize microbe-associated molecular patterns (MAMPs) to initiate MAMP-triggered immunity (Boller and Felix 2009). Other RLK, such as BAK1/ SERK3 and SOBIR1, function as central components in regulation of various receptor-mediated signaling processes (Chinchilla et al. 2009; Liebrand et al. 2013). More insight into the function of a wider and more diverse range of RLK would help to better understand how plants interact with their environment.

Based on the divergent extracellular domains, RLK can be divided into different classes. One of the largest RLK classes in plants comprises the L-type lectin receptor kinases (LecRK). LecRK are characterized by an extracellular lectin domain structurally resembling soluble lectins of leguminous plants (Hervé et al. 1996). In Arabidopsis, 45 LecRK have been identified that were divided into nine clades (i.e., clade I to IX) and seven singletons (Bouwmeester and Govers 2009). Given this large number, LecRK are expected to play diverse roles in plant development and defense. Thus far, functional characterization has been limited to a few Arabidopsis LecRK. For example, LecRK-IV.2 (alias SGC lectin RLK) was suggested to be essential for proper pollen development because a T-DNA insertion mutant showed deformed and collapsed pollen grains (Wan et al. 2008). LecRK-V.2 (alias LecRK-b2) and three LecRK from clade VI (previously named LecRK4.1, LecRK4.2, and LecRK4.3) were shown to be involved in regulating abscisic acid (ABA) response during seed germination (Deng et al. 2009; Xin et al. 2009). Later studies also demonstrated a role for LecRK in plant immunity. LecRK-I.9, a mediator of plant cell wall-plasma membrane integrity (Gouget et al. 2006), was shown to be crucial for Phytophthora resistance in Arabidopsis (Bouwmeester et al. 2011). More recently, the same LecRK was identified as the first ATP receptor in plants and shown to be required for ATP-mediated responses (Choi et al. 2014). As yet, it is not clear if the function of LecRK-I.9 as a Phytophthora resistance component is linked to its function as ATP receptor. Two other LecRK, LecRK-V.5 and LecRKVI.2, were shown to be involved in bacterial resistance by regulating stomatal immunity (Desclos-Theveniau et al. 2012; Singh et al. 2012). The latter was found to associate with the bacterial flagellin-receptor FLS2 and to be a positive regulator of the flagellin-mediated defense responses (Huang et al. 2014). LecRK-IV.3 (alias AtLPK1) might have a role in resistance to fungal pathogens because LecRK-IV.3 overexpression in Arabidopsis resulted in increased resistance to Botrytis cinerea (Huang et al. 2013). For several other LecRK, a func- 
tion in plant growth or stress response was anticipated based on in silico expression analysis (Bouwmeester and Govers 2009) but, as yet, experimental evidence for this is lacking.

The objective of the current work was to elucidate the biological function of individual LecRK in Arabidopsis. LecRK TDNA insertion lines were obtained and analyzed for phenotypic changes i) in plant development under normal conditions or abiotic stress and ii) upon exposure to pathogens. The phenotypic data were then integrated with in silico expression data with the aim to uncover potential associations between LecRK gene expression and biological function. In addition, LecRK$V .5$, of which a mutant showed altered disease phenotypes, was selected for more detailed study to validate its function.

\section{RESULTS}

\section{Assembling the collection of Arabidopsis LecRK T-DNA insertion lines.}

The T-DNA Express database of the SALK Institute Genome Analysis Laboratory was used to search for T-DNA insertion lines for all 45 LecRK (Table 1). Preferably, homozygous lines bearing T-DNA insertions within the coding regions were selected to enhance the possibility of complete disruption of gene function. If not available, homozygous lines with insertions in the promoter (five lines), $5^{\prime}$ untranslated region (UTR) (14 lines), 3'UTR (two lines), or segregating lines were obtained. In total, we obtained 62 T-DNA insertion lines covering 41 LecRK, all in a Col-0 background. The seed of the only T-DNA insertion line available for LecRK-IV.3 (i.e., SAIL 529_D07) did not geminate, thereby reducing the number of lines to 61 , covering 40 LecRK. Of these 61, 52 were indicated as homozygous lines and nine as segregating lines. To confirm this, we performed polymerase chain reaction (PCR) analysis and found that 42 of the 52 lines were indeed homozygous with correct T-DNA insertion sites. Of the remaining 10 lines, eight were found to be segregating at the site of the T-DNA insertion and two (i.e., SALK_053703C and SALK_019496C) did not show a T-DNA insertion in the anticipated target gene (i.e., LecRK-IV.1), indicating that these lines are either untransformed Col-0 or contain a T-DNA insertion elsewhere in the genome. Therefore, in total, we ended up with 17 segregating lines. These were propagated and the offspring was genotyped to select the homozygous ones. For five lines, however, we were not able to detect any homozygous offspring among over 60 plants tested for each individual line. This was the case for the single T-DNA insertion line that was available for LecRKII.2 as well as for the two lines mutated in LecRK-V.6 and the two in LecRK-S.5. The consistency among the two independent LecRK-V.6 lines and the two independent LecRK-S.5 lines suggests that a complete knockout of these genes is lethal, raising the possibility that these LecRK have a crucial role in development. In total, 54 lines representing 36 individual LecRK were confirmed as homozygous T-DNA insertion lines and used for further analysis (Table 1).

\section{Arabidopsis LecRK T-DNA insertion lines showed no phenotypic changes in growth and development.}

To determine whether LecRK are involved in plant growth and development, LecRK T-DNA insertion lines were grown in soil and analyzed for phenotypic changes in rosette size, inflorescence emergence, and flower and silique morphology for up to 8 weeks. In addition, seed were grown in vitro on vertical 0.5 Murashige Skoog (MS) plates to monitor shoot and root growth. Under these conditions, no consistent growth alterations were observed compared with Col-0 (Table 1). Previously, Wan and associates (2008) described that a homozygous mutant with two insertions in the region encoding the lectin do- main of LecRK-IV.2 developed abnormal siliques and they concluded that LecRK-IV.2 is required for proper pollen development. The T-DNA insertion line lecrk-IV.2 that we analyzed harbors a T-DNA insertion in the 5' UTR, at approximately 70 bp before the start codon. Because this line showed no defects in silique development, we analyzed the LecRK-IV.2 expression by quantitative reverse-transcriptase (qRT)-PCR and found that the transcript level is only slightly reduced when compared with Col-0 (approximately 40\%) (Supplementary Fig. S1A and B). This reduction is apparently not sufficient to disturb normal silique development. Unfortunately, the mutant analyzed by Wan and associates (2008) is no longer available in public resources, thus hampering further functional analysis of LecRK-IV.2.

\section{Response of LecRK T-DNA insertion lines to abiotic stress.}

To evaluate the role of $L e c R K$ in resistance to abiotic stresses, the LecRK T-DNA insertions lines were grown on 0.5 MS medium supplemented with $100 \mathrm{mM} \mathrm{NaCl}$ or $200 \mathrm{mM}$ mannitol. Plants were checked for changes in rosette size and growth of shoots and roots. No consistent differences were observed between any of the LecRK T-DNA insertion lines and Col-0. Previously, Deng and associates (2009) observed that a line with a T-DNA insertion in LecRK-V.2 showed enhanced tolerance to $\mathrm{NaCl}$ and mannitol and developed longer primary roots than Col-0, and concluded that LecRK-V.2 is involved in salt and osmotic stress. In our study, lecrk-V.2, a T-DNA insertion line with an insertion in the $3^{\prime}$ UTR, approximately $60 \mathrm{bp}$ after the stop codon, showed growth similar to that of Col-0 upon treatment with $\mathrm{NaCl}$ or mannitol, also when treated using exactly the same conditions as described by Deng and associates (2009) (Table 1; Supplementary Fig. S2A and B). To find a reason for this discrepancy, we determined the LecRK-V.2 expression in lecrk-V.2 and Col-0 by qRT-PCR. Remarkably, the transcript level in lecrk-V.2 was higher than in Col-0, instead of lower. Possibly the T-DNA insertion in the $3^{\prime}$ UTR in one way or another stabilizes the mRNA and this may explain why we did not find defects in salt and osmotic stress responses. T-DNA insertion line lecrk-V.2 was excluded from further analysis.

\section{Response of LecRK T-DNA insertion lines to Phytophthora pathogens.}

Previously, LecRK-I.9 was reported as a novel resistance component against Phytophthora brassicae in Arabidopsis (Bouwmeester et al. 2011). To determine the involvement of other LecRK in Phytophthora resistance, we performed infection assays on the LecRK T-DNA insertion lines with $P$. brassicae, $P$. capsici, and $P$. parasitica. Recently it was reported that the latter two are able to infect Arabidopsis (Wang et al. 2011, 2013).

In order to identify LecRK contributing to Phytophthora resistance, infection assays were performed with isolates that are not able to infect Arabidopsis accession Col-0 (i.e., P. brassicae $\mathrm{HH}$ and $P$. capsici LT123). As shown by Bouwmeester and associates (2011), isolate HH can successfully infect LecRKI.9 mutants. Inoculation of lecrk-I.9-1 and lecrk-I.9-2 with $P$. brassicae $\mathrm{HH}$ resulted in water-soaked lesions at 4 days after inoculation (dpi), with a mean disease severity index (DSI) of 2.2 and 2.4, respectively (Supplementary Fig. S3A). P. capsici LT123 caused similar disease symptoms on these mutants and, at $4 \mathrm{dpi}$, the mean DSI was 2.0 and 1.9 , respectively. This shows that LecRK-I.9 also contributes to resistance against $P$. capsici.

Inoculation of all available LecRK T-DNA insertion lines with $P$. brassicae $\mathrm{HH}$ and $P$. capsici LT123 resulted in evident disease symptoms on several lines. For example, lecrk-S.1, 
which contains a T-DNA insertion in the region encoding the extracellular lectin domain and has a $90 \%$ reduction in LecRKS. 1 expression level (Fig. 1A and B), showed a mean DSI of 2.5 and 2.1 with $\mathrm{HH}$ and LT123, respectively (Fig. 1C). In total, 19 T-DNA insertion lines representing 13 LecRK from different clades showed a significantly higher DSI at 4 dpi than Col-0 (Table 1). Of these 13 LecRK, three are from clade V, two from clade IX, and four are singletons. The four remaining LecRK are from clades I, III, IV, and VIII, respectively. For four of 13, two T-DNA insertion lines are available which both showed enhanced disease severity when challenged with Phytophthora spp., with at least one of the two having an insertion in the coding region (Table 1). For two other LecRK for which two or more T-DNA insertion lines are available, T-DNA insertion lines with different insertion sites showed discrepancy in susceptibility. In the case of LecRK-VIII.2, with three lines having insertions in the coding region, only lecrk-VIII.2-3 developed rapid spreading lesions, resulting in a DSI $\geq 2$ on approximately $80 \%$ of the inoculated leaves at 4 dpi. The other two lines, lecrk-VIII.2-1 and lecrk-VIII.2-2, also showed disease symptoms but the disease severity was less pronounced (Table 1). In the case of LecRK-S.7, the two T-DNA insertion lines showed opposite responses. Line lecrk-S.7-1 is fully resistant to both $P$. brassicae $\mathrm{HH}$ and $P$. capsici LT123, whereas lecrk-S.7-2 showed clear disease symptoms with a mean DSI of 2.0 and 1.9, respectively (Table 1). Both lines have the TDNA insertion in the $5^{\prime}$ UTR but at different positions. Expression analysis revealed that the difference in phenotype between lecrk-S.7-1 and lecrk-S.7-2 correlates with LecRK-S.7 expression levels. In lecrk-S.7-2, expression is reduced compared with Col-0 whereas, in lecrk-S.7-1, it is increased (Supplementary Fig. S4B). For the seven remaining LecRK of which T-DNA

Table 1. Overview of the clades and members of the LecRK gene family in Arabidopsis, the T-DNA insertion lines used in this study, and their phenotypes in response to pathogen infection and abiotic stress

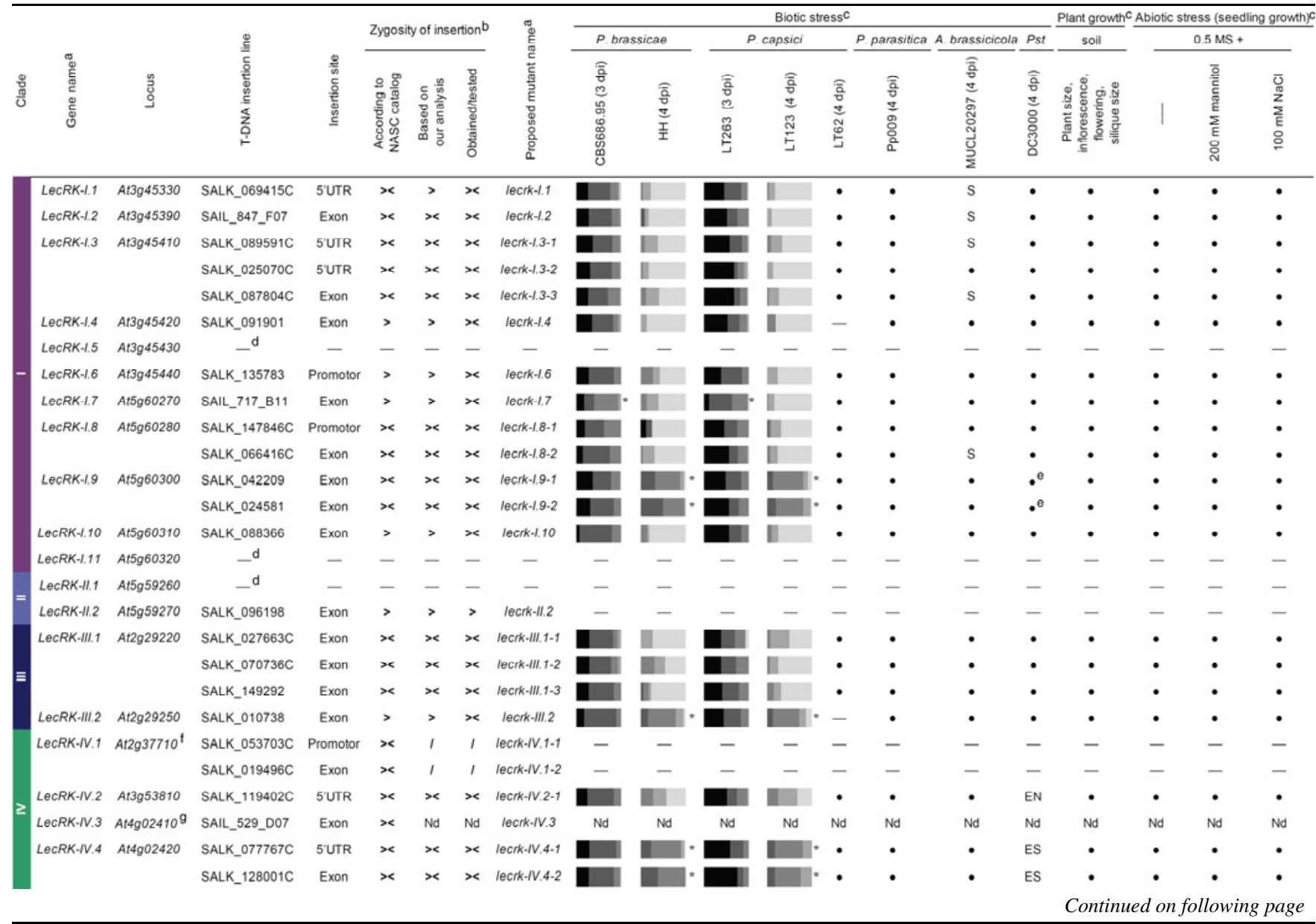

${ }^{a}$ Alternative gene names and alternative mutant names are provided in Supplementary Table S1.

${ }^{\mathrm{b}}><$ : homozygous; >: segregating; /: no insertion in the gene under study.

${ }^{c}$ The response to Phytophthora brassicae and P. capsici is depicted by bars that show the disease severity index (DSI) obtained from 20 to 40 inoculated leaves in three independent experiments. The DSI scale ranges from 0 (light gray) to 4 (dark gray). An asterisk (*) indicates T-DNA insertion lines showing significantly higher or lower DSI as compared with Col-0, based on a $t$ test, $P<0.05$. Response to infection with the remaining pathogens, plant growth, and response to abiotic stress are depicted by a black dot $(\bullet)$ when there is no difference compared with Col-0 and by S (susceptible), R (resistant), EN (enhanced necrotic symptoms but no increased bacterial proliferation), or ES (enhanced necrotic symptoms and increased bacterial proliferation) to indicate the disease phenotype. Pst = Pseudomonas syringae pv. tomato. Infection assays with P. capsici LT62 were performed once while infection assays with all other pathogens were repeated at least three times.

d No T-DNA insertion lines available.

${ }^{\mathrm{e}}$ With 10-fold higher inoculation densities, lecrk-I.9-1 and lecrk-I.9-2 show enhanced susceptibility to Pseudomonas syringae pv. tomato, as reported by Bouwmeester and associates (2011).

f SALK_053703C and SALK_019496C were indicated as lines with T-DNA insertions in At2g37710, but in our analysis, this could not be confirmed.

g The obtained SAIL_529_D07 line did not germinate.

${ }^{\mathrm{h}}$ Similar results were obtained as described by Desclos-Theveniau and associates (2012) and Singh and associates (2012). 
insertion lines showed a change in phenotype in the Phytophthora disease assays, only a single T-DNA insertion line was available, with six (i.e., lecrk-III.2, lecrk-V.4, lecrk-V.5, lecrkV.7, lecrk-IX.2, lecrk-S.1, and lecrk-S.6) having a T-DNA insertion in the coding region and one (i.e., lecrk-V.5-3) in the $5^{\prime}$ UTR (Table 1).

When challenging the LecRK T-DNA insertion lines with Phytophthora isolates that are less virulent (in this case, $P$. capsici LT62 and P. parasitica Pp009), none of the LecRK TDNA insertion lines showed lesions (Table 1). They remained resistant, similar to Col-0. This suggests that these LecRK are not essential to stop less virulent isolates but might function as an extra barrier for more virulent isolates.

To investigate the potential role of $L e c R K$ in suppressing Phytophthora resistance, LecRK T-DNA insertion lines were challenged with Phytophthora isolates that are capable of infecting Col-0 (i.e., P. brassicae CBS686.95 and P. capsici
LT263). Out of all tested LecRK T-DNA insertion lines, only lecrk-I.7, with an insertion in the region encoding the kinase, showed slightly smaller lesions when compared with Col-0 (Fig. 1D). All the other T-DNA insertion lines showed disease severity comparable with or slightly higher than Col-0 (Table 1).

\section{Response of LecRK T-DNA insertion lines}

to Pseudomonas syringae and Alternaria brassicicola.

Previously, LecRK-VI.2 was reported to play a role in resistance to bacterial pathogens (Singh et al. 2012). To determine the involvement of other LecRK in bacterial resistance, we performed infection assays with Pseudomonas syringae DC3000. Upon spray inoculation, T-DNA insertion lines of eight LecRK showed enhanced yellowing and necrosis at $4 \mathrm{dpi}$ when compared with Col-0 (Table 1; Fig. 2A). For three of these, two T-DNA insertion lines are available which both showed enhanced symptoms when challenged with Pseudomo-

Table 1. Continued from previous page

\begin{tabular}{|c|c|c|c|c|c|c|c|c|c|c|c|c|c|c|c|c|c|c|c|}
\hline \multirow[b]{3}{*}{ 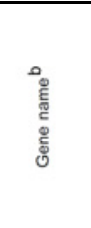 } & \multirow[b]{3}{*}{$\stackrel{n}{\overrightarrow{3}}$} & \multirow{3}{*}{ 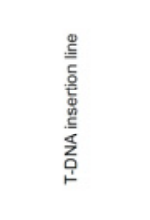 } & \multirow[b]{3}{*}{ 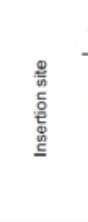 } & \multirow{2}{*}{\multicolumn{3}{|c|}{ Zygosity of insertion ${ }^{c}$}} & \multirow{3}{*}{ 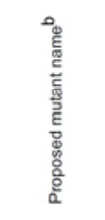 } & \multicolumn{8}{|c|}{ Biotic stress } & \multirow{3}{*}{$\frac{\frac{\text { Plant growth }}{\text { soil }}}{\text { soll }}$} & \multirow{2}{*}{\multicolumn{3}{|c|}{$\begin{array}{l}\text { Abiotic stress (seedling gromh) } \\
0.5 \mathrm{MS}+ \\
\end{array}$}} \\
\hline & & & & & & & & \multicolumn{2}{|c|}{ P. brassicaed } & \multicolumn{2}{|c|}{ P. capsicid } & & \multirow{2}{*}{ 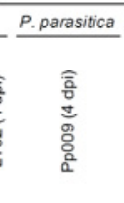 } & \multirow{2}{*}{$\frac{\text { A. brassicicola }}{\text { - }}$} & \multirow[b]{2}{*}{ 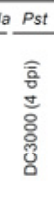 } & & & & \\
\hline & & & & 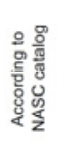 & 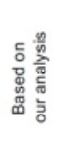 & 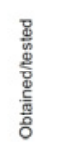 & & 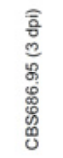 & 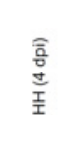 & $\widehat{\widehat{\widehat{a}}}$ & 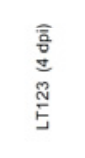 & $\begin{array}{l}\text { 言 } \\
\text { 产 }\end{array}$ & & & & & | & 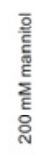 & 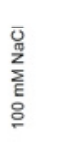 \\
\hline LecRK-V. 1 & At1g70110 & SALK_071152 & Promotor & $x$ & $x$ & $x$ & lecrk-V.1 & & 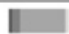 & & ! & - & - & . & • & . & . & . & . \\
\hline LecRK-V.2 & At1g70130 & SALK_014678C & 3'UTR & $x$ & $x$ & $x$ & lecrk-V.2 & & - & & - & - & - & - & - & - & - & - & - \\
\hline LeCRK-V.3 & At2g 43690 & SALK_013197C & 5'UTR & $x$ & $x$ & $x$ & lecrk-V.3 & & 口 & & $\square$ & • & - & - & • & - & - & - & - \\
\hline LeCRK-V. 4 & At2g 43700 & SALK_119422C & Exon & $>$ & $x$ & $x$ & lecrk-V.4 & & $\square$ & & 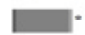 & - & - & - & EN & - & . & - & - \\
\hline LeCRK-V. 5 & At3959700 & SALK_133163C & ${ }^{5}$ UTR & $x$ & $x$ & $x$ & lecrk-V.5-3 & & - & & $\square$ & - & - & - & $R^{0}$ & - & - & - & - \\
\hline LecRK-V. 6 & At3959730 & SALK_009623C & Exon & $x$ & $>$ & $>$ & lecrk-V.6-1 & & - & & - & - & - & - & - & - & - & - & - \\
\hline & & SALK_083045C & Exon & $x$ & $>$ & $>$ & lectrk-V.6-2 & & - & & - & - & - & - & - & - & - & - & - \\
\hline LeCRK-V. 7 & At3959740 & SALK_151041 & Exon & $>$ & $>$ & $x$ & lecrk-v.7 & & & & 口 & - & - & - & EN & - & - & - & - \\
\hline LecRK-V. 8 & At3g59750 & WiscDsLox381A8 & Promotor & $x$ & $x$ & $x$ & lecrk-V. 8 & & 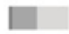 & & 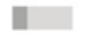 & - & - & - & • & - & - & - & - \\
\hline LeCRK-V.g & At 4929050 & SALK_092640C & 5'UTR & $x$ & $x$ & $x$ & lectrk-V.9-1 & & I & & 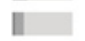 & - & - & - & - & - & - & - & - \\
\hline & & SALK_009531C & Exon & $x$ & $x$ & $x$ & lecrk-V.9-2 & & 口 & & I & - & • & - & • & - & • & - & - \\
\hline & & SAIL_917_A03 & Exon & $x$ & $x$ & $x$ & lectk-v.9.3 & & I & & I & • & - & • & - & - & - & - & - \\
\hline LeCRK-VI.1 & At3g08870 & SALK_058958C & Exon & $>$ & $>$ & $x$ & lecrk-VI.1 & & $\mathbf{\square}$ & & 口 & - & - & - & • & - & • & - & - \\
\hline LeCRK-VI.2 & At5go1540 & SALK_877782 & Exon & $x$ & $x$ & $x$ & lecrk-VI.2-1 & & ㅁ & & 口 & - & - & - & ES & - & - & - & - \\
\hline & & SALK_ 070801 & Exon & $>$ & $>$ & $><$ & lecrk-VI.2-2 & & 口 & & 口 & - & - & - & $\mathrm{ES}^{\mathrm{i}}$ & - & $\cdot$ & - & - \\
\hline LeCRK-VI.3 & At5g01550 & SALK_151042C & 5'UTR & $x$ & $x$ & $x$ & lecrk-VI.3-1 & & 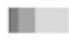 & & I & • & • & - & - & - & - & - & - \\
\hline & & SALK_108000C & Exon & $x$ & $x$ & $x$ & lecrk-VI.3.2 & & $\mathbf{D}$ & & 口 & - & • & • & - & - & - & - & - \\
\hline LeCRK-VII.4 & At 5901560 & SALK_026891C & ${ }^{5}$ UTRR & $>$ & $x$ & $>$ & lecrk-VI.4-1 & & I & & 口 & - & - & - & • & - & • & - & - \\
\hline & & SALK_122197 & Exon & $>$ & $>$ & $x$ & lecrk-VI.4-2 & & [ & & \| & - & - & - & • & - & - & - & - \\
\hline LecRK-VII.1 & At4904960 & SALK_093876C & Exon & $x$ & $x$ & $x$ & lecrk-VII.1 1 & & a & & I & - & • & • & • & - & • & - & - \\
\hline LeCRK-VII. 2 & At4g28350 & SALK_141841C & 3'UTR & $>$ & $>$ & $x$ & lecrk-VII.2 & & D. & & 口 & - & - & - & - & - & - & - & - \\
\hline LeCRK-VIII. 1 & At3953380 & SALK_092365C & Exon & $x$ & $x$ & $>$ & lecrk-VIII.1-1 & & I & & 口 & - & • & • & • & - & - & - & - \\
\hline & & SALK_056492C & Exon & $x$ & $x$ & $x$ & lecrk-VIII.1-2 & & 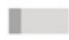 & & 【 & - & • & • & - & - & - & • & - \\
\hline LeCRK-VIII. 2 & At5g03140 & SALK_051706C & Exon & $x$ & $>$ & $><$ & lecrk-VIII.2-1 & & 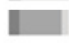 & & $\square$ & - & • & • & - & - & - & - & - \\
\hline & & SALK_053278C & Exon & $x$ & $>$ & $x$ & lecrk-VIII.2-2 & & 口 & & 口 & - & - & - & • & - & - & - & - \\
\hline & & SALK_132863C & Exon & $x$ & $x$ & $x$ & lecrk-VIII. 2-3 & & & & L & - & - & - & • & - & • & - & - \\
\hline LeCRK-IX.1 & At5g10530 & SALK_127554C & Exon & $>$ & $x$ & $x$ & leck-1X.1-1 & & & & 世 & - & - & - & - & - & $\cdot$ & • & - \\
\hline & & SALK_042414C & Exon & $x$ & $x$ & x & lecrk-1X.1-2 & & & & 口. & - & - & - & • & - & - & - & . \\
\hline LeCRK-IX.2 & At5g65600 & SALK_111817 & Exon & $x$ & $x$ & $x$ & lecrk-1X.2 & & 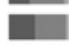 & & ए. & - & - & - & - & - & - & - & - \\
\hline LeCRK-S.1 & Attg15530 & SALK_033248C & Exon & $x$ & $x$ & » & lecrk-S.1 & & 口 & & = & - & - & - & ES & - & - & - & - \\
\hline LecRK-S. 2 & At2g32800 & SAIL_510_C08 & Exon & $>$ & $>$ & $\gg$ & lecrk-S.2 & & ㅁ & & $\mathbf{D}$ & - & - & - & - & - & - & - & - \\
\hline LecRK-S. 3 & At3g 46760 & $-{ }^{e}$ & - & - & - & - & - & & - & - & - & - & - & - & - & - & - & - & - \\
\hline LeCRK-S. 4 & At3a55550 & SALK_059967C & $5^{\prime}$ UTR & $>$ & $x$ & $><$ & lecrk-S.4-1 & & 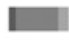 & & 口. & • & - & • & ES & - & $\cdot$ & - & - \\
\hline & & SAlL_795_F11 & Exon & $>$ & $x$ & $><$ & lecrk-S.4-2 & & 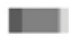 & & $\square$ & - & - & - & ES & - & • & • & - \\
\hline LecRK-S. 5 & At5g06740 & SALK_088076C & 5'UTR & $x$ & $>$ & $>$ & lecrk-S.5.1 & - & - & - & - & - & - & - & - & - & - & - & - \\
\hline & & SAlL_1278_B07 & Exon & $>$ & $>$ & $>$ & lectrk-S.5.2 & - & - & - & - & - & - & - & - & - & - & - & - \\
\hline LecRK-S. 6 & At5g42120 & SALK_151974C & Exon & $x$ & $>$ & $x$ & lecrk-S.6 & & 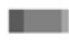 & & 口. & - & - & • & EN & - & - & - & - \\
\hline LecRK-S.7 & At5g55830 & SALK_140480C & 5'UTR & $x$ & $x$ & $x$ & lecrk-S.7-1 & & ए & & I & - & - & - & • & - & • & • & - \\
\hline & & SALK_008479C & 5'UTR & $x$ & $x$ & $x$ & leckr-S.7.2 & & 口 & & 口. & - & - & • & • & • & • & • & - \\
\hline Col-0 & & & & & & & & & 口 & & & - & - & - & • & - & • & - & - \\
\hline
\end{tabular}


nas syringae DC3000, with at least one of the two having an insertion in the coding region (Table 1). To determine to what extent the visible symptoms are correlated with enhanced Arabidopsis susceptibility, bacterial colonization was quantified in rosette leaves. In line with Singh and associates (2012), we detected significantly higher bacterial titers in lecrk-VI.2-2 compared with Col-0 (Fig. 2B). Also, in lesions on the two T-DNA insertion lines from LecRK-IV.4, the one from LecRK-S.1, and two from LecRK-S.4, significantly higher bacterial titers were detected and this was reproducible (Fig. 2B). Thus, these T-DNA insertion lines were scored as enhanced susceptibility (ES). The phenotype of the remaining four T-DNA insertion lines (i.e., lecrk-IV.2, lecrk-V.4, lecrk-V.7, and lecrk-S.6) was scored as enhanced yellowing and necrosis $(\mathrm{EN})$ rather than ES (Table 1). This is because the bacterial titers were not consistently higher in all assays, despite the fact that the enhanced susceptibility phenotypes were reproducible.

To determine the potential involvement of LecRK in nonhost resistance, we tested the response of the LecRK T-DNA insertion lines to Alternaria brassicicola, a fungal pathogen that is not pathogenic on Col-0 but is capable of causing disease symptoms on the camalexin-deficient mutant pad3 (Van Wees et al. 2003). The majority of the LecRK T-DNA insertion lines showed no visible symptoms up to 5 dpi. Five T-DNA insertion lines, however, representing four clade I LecRK, showed a slight gain of susceptibility (Table 1; Fig. 2C). Two of three LecRK-I.3 T-DNA insertion lines showed the phenotype, one with an insertion in the exon (lecrk-I.3-3) and the other in the
5' UTR (lecrk-I.3-1). The third (i.e., lecrk-I.3-2), also with a 5' UTR insertion, was not infected. Also, in the case of LecRKI.8, the exon mutant (lecrk-I.8-2) showed gain of susceptibility but not the T-DNA insertion line, with a $5^{\prime}$ UTR insertion (lecrk-I.8-1).

\section{LecRK-V.5 plays a dual role in resistance}

to Pseudomonas syringae and Phytophthora pathogens.

Our infection assays revealed that the mutant lecRK-V.5-3 showed gain of susceptibility to Phytophthora brassicae $\mathrm{HH}$ and $P$. capsici LT123 (Table 1; Fig. 3A to C). In contrast, Desclos-Theveniau and associates (2012) reported that mutants of LecRK-V.5 showed enhanced resistance toward DC3000. Because of these opposite phenotypes with respect to different pathogens, we decided to investigate the function of LecRKV.5 in more detail.

Gain of Phytophthora susceptibility in lecrk-V.5-3 points to a role of LecRK-V.5 in Phytophthora resistance. To confirm this, we generated transgenic Arabidopsis lines overexpressing LecRK-V.5 in a Col-0 background. Two lines (i.e., 35S-LecRK$V .5-1 / 2$ ) with constitutive and strongly enhanced LecRK-V.5 expression were selected (Fig. 3B). Neither of the $35 S$-LecRKV.5 lines showed any visible developmental defect. Inoculation of Col-0 with $P$. brassicae CBS686.95 and P. capsici LT263 resulted in almost fully colonized leaves at 3 dpi (Fig. 3D). In contrast, the two $35 S$-LecRK-V.5 lines showed reduced lesion sizes, with a DSI of approximately 2.0 (Fig. 3D). To determine whether the reduced disease severity on the LecRK-V.5 overex-
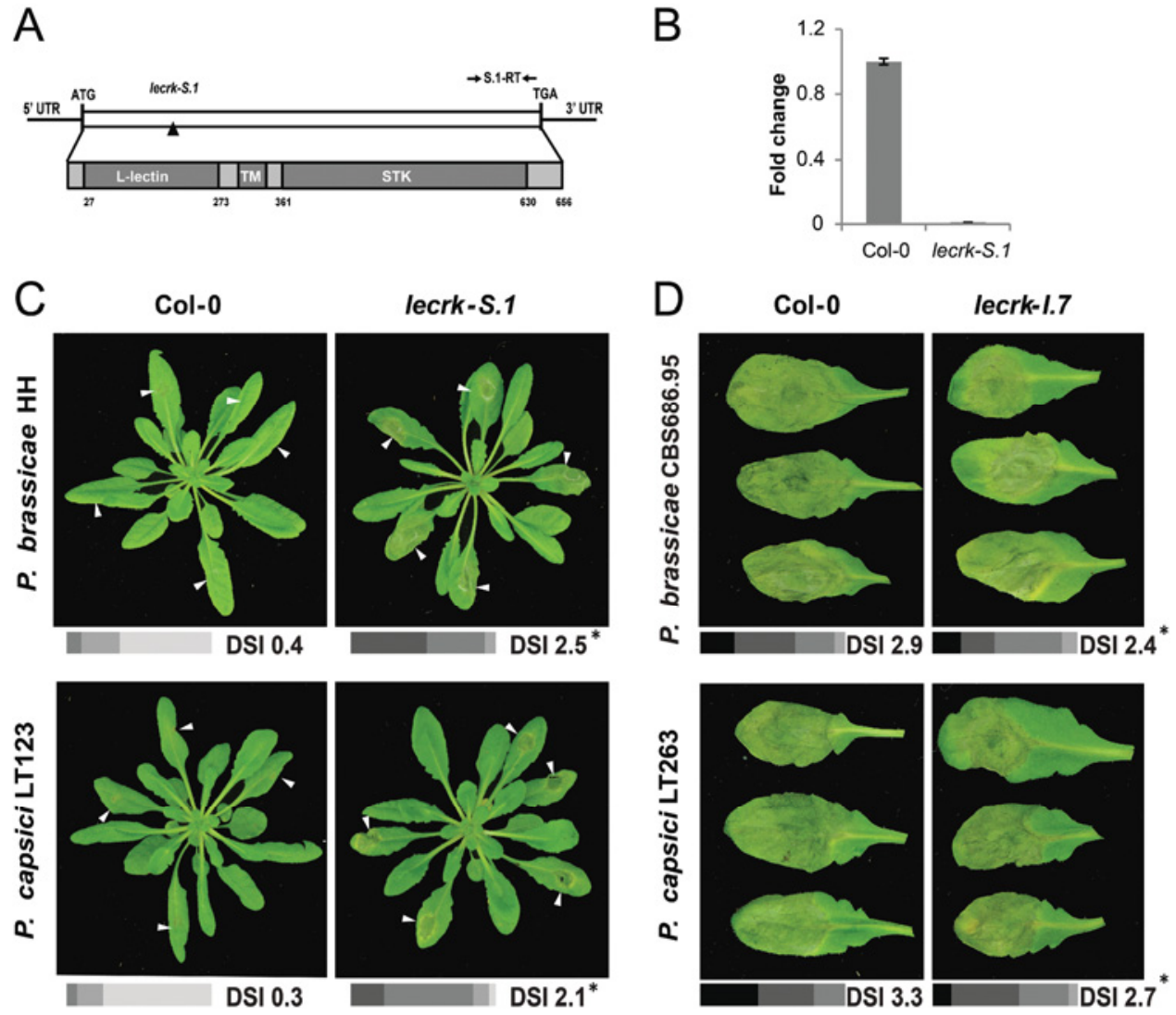

Fig. 1. Response of Arabidopsis LecRK T-DNA insertion lines to infection by Phytophthora spp. A, Schematic model representing LecRK-S.1. The black arrowhead points to the T-DNA insertion site in mutant lecrk-S.1 while the head-to-head arrow pairs indicate the position of the primers (S.1-RT) used for quantitative reverse-transcriptase polymerase chain reaction (qRT-PCR). B, Relative quantification of LecRK-S.1 transcript levels in Col-0 and lecrk-S.1 by qRT-PCR. C, Disease symptoms on Col-0 and lecrk-S.1 at 4 days after plug-inoculation (dpi) with $P$. brassicae HH and P. capsici LT123. White arrowheads point to inoculated leaves. Each bar represents the disease severity index (DSI) obtained from at least 30 leaves in three independent experiments, with the number showing the average DSI and an asterisk $(*)$ indicating a significant difference $(P<0.05)$ according to a $t$ test. D, Disease symptoms on T-DNA insertion line lecrk-I.7 and Col-0 inoculated with $P$. brassicae CBS686.95 and $P$. capsici LT263 at 3 dpi; * indicates significant difference in DSI $(P<0.05)$ according to a $t$ test. 
pression lines is indeed correlated with enhanced resistance, Phytophthora biomass was quantified by qRT-PCR in leaves inoculated with $P$. capsici LT263 at 3 dpi. This showed that the $P$. capsici biomass in the $35 S-L e c R K-V .5$ lines was reduced by at least 50\% compared with Col-0 (Fig. 3E).

To confirm the role of LecRK-V.5 in resistance to DC3000 as postulated by Desclos-Theveniau and associates (2012), we compared the phenotype of lecrk-V.5-3 with the phenotypes of the previously analyzed mutants lecrk-V.5-1 and lecrk-V.5-2 Similar to lecrk-V.5-1 and lecrk-V.5-2, lecrk-V.5-3 showed fewer necrotic symptoms and reduced bacterial proliferation compared with Col-0 (Fig. 3F and G). Moreover, both $35 \mathrm{~S}$ LecRK-V.5 lines showed enhanced susceptibility to DC3000, with increased necrosis and higher bacterial titers than Col-0 (Fig. 3F to G). Taken together, these results demonstrate that LecRK-V.5 has a dual role in regulating defense to different pathogens.

\section{Are there associations between LecRK mutant phenotypes and LecRK expression profiles?}

Expression profiling analysis has been widely used to predict potential biological functions of genes (Fukushima et al. 2012; Li et al. 2006). Here, we explored the transcriptome-tophenotype relations by comparing LecRK expression profiles retrieved from publicly available microarray data with the mutant phenotypes obtained in this study (Fig. 4). Because there is no genome-wide expression data available from Arabidopsis inoculated with $P$. brassicae or $P$. capsici, we used the expression data derived from Arabidopsis leaves inoculated with $P$. infestans, a Phytophthora sp. that is not capable of infecting Arabidopsis (Huitema et al. 2003). The 13 LecRK, of which T-DNA insertion lines showed compromised resistance to $P$. brassicae $\mathrm{HH}$ and $P$. capsici $\mathrm{LT} 123$, were found to be differen- tially expressed during $P$. infestans infection (Fig. 4A). LecRK$I X .2$ was highly induced and the expression level was increased up to 50 -fold ( $\log _{2}$ value of 5.7$)$ at $6 \mathrm{~h}$ postinfection (hpi). LecRK-I.9, LecRK-V.5, and LecRK-V.7 showed induced expression with a maximum of fourfold-increase, whereas the other nine LecRK were either hardly expressed or even downregulated during infection. The same holds for LecRK-I.7, the only LecRK of which the mutant showed slightly enhanced Phytophthora resistance.

The nine LecRK of which T-DNA insertion lines showed altered phenotypes in response to DC3000 (EN, ES, or resistant $[\mathrm{R}]$ ) were all downregulated at one or more time points after inoculation with DC3000 (Fig. 4A). The five LecRK with T-DNA insertion lines showing significantly altered bacterial colonization (ES or R) were downregulated at $12 \mathrm{hpi}$, with $\log _{2}$ values ranging from -0.7 to -1.4 .

Despite the fact that several LecRK were differently expressed in various tissues and in response to $\mathrm{NaCl}$ or mannitol treatment, none of the tested T-DNA insertion lines showed consistent phenotypic changes during growth and development or when exposed to abiotic stress. Hence, it is difficult to reconcile the biological function of these LecRK with expression profiles. However, lack of phenotypic changes in mutants does not exclude the possibility that certain LecRK have a function in these processes. It could well be that functional redundancy masks the phenotypes caused by a single gene disruption.

Genes that exhibit similar expression patterns are often expected to be involved in the same biological processes (Eisen et al. 1998; Fukushima et al. 2012). We computed coexpression profiles of all LecRK in leaves inoculated with $P$. infestans and DC3000 over time. In order to easily visualize LecRK expression patterns, expression levels ( $\log _{2}$ values) were normalized by Z-score transformation (Cheadle et al. 2003). LecRK
A

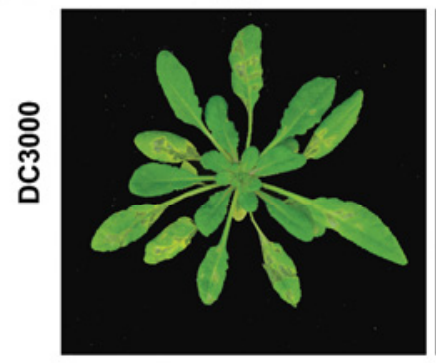

B

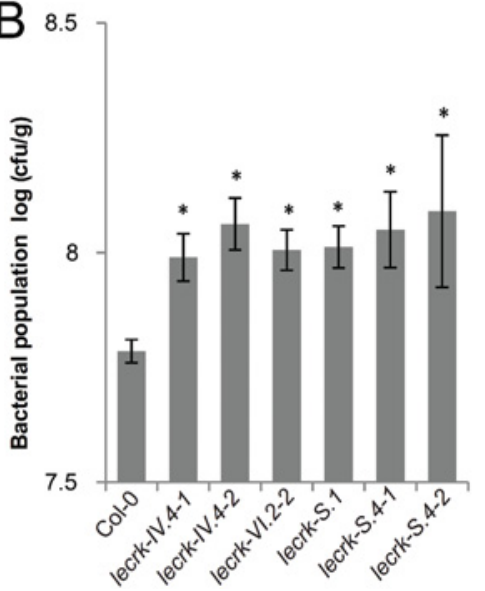

lecrk-VI.2-2

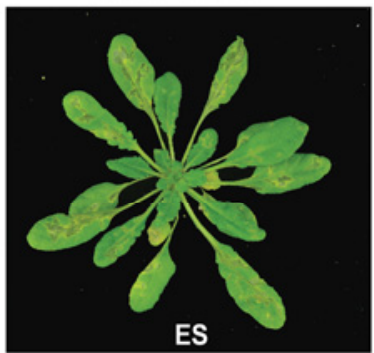

C
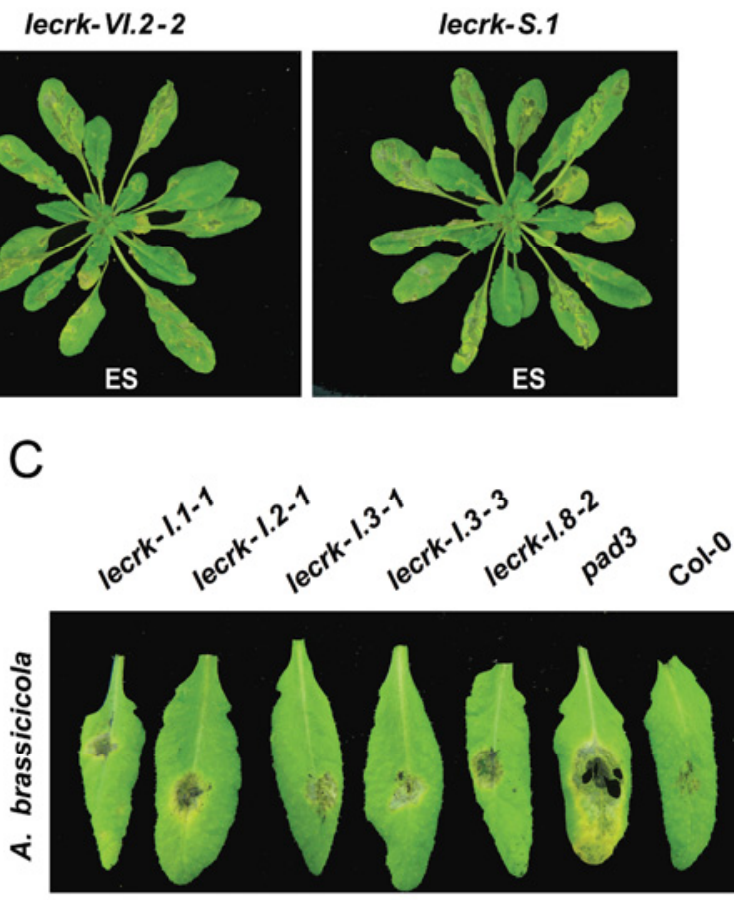

Fig. 2. Response of Arabidopsis LecRK T-DNA insertion lines to infection by Pseudomonas syringae and Alternaria brassicicola. A, Disease symptoms on Col-0, lecrk-S.1, and lecrk-VI.2-2 4 days after inoculation (dpi) with P. syringae DC3000 $\left(10^{8} \mathrm{CFU} \mathrm{ml}^{-1}\right)$. B, Bacterial growth in Col-0 and various LecRK TDNA insertion lines at 3 dpi. Bars depict mean bacterial titers of four plants ( \pm standard deviation); * indicates $P<0.05$ according to a $t$ test for comparisons between Col-0 and LecRK T-DNA insertion lines. Experiments were repeated three times with similar results. C, Disease symptoms on Col-0, pad 3 and various LecRK T-DNA insertion lines 5 dpi with A. brassicicola MUCL20297. 

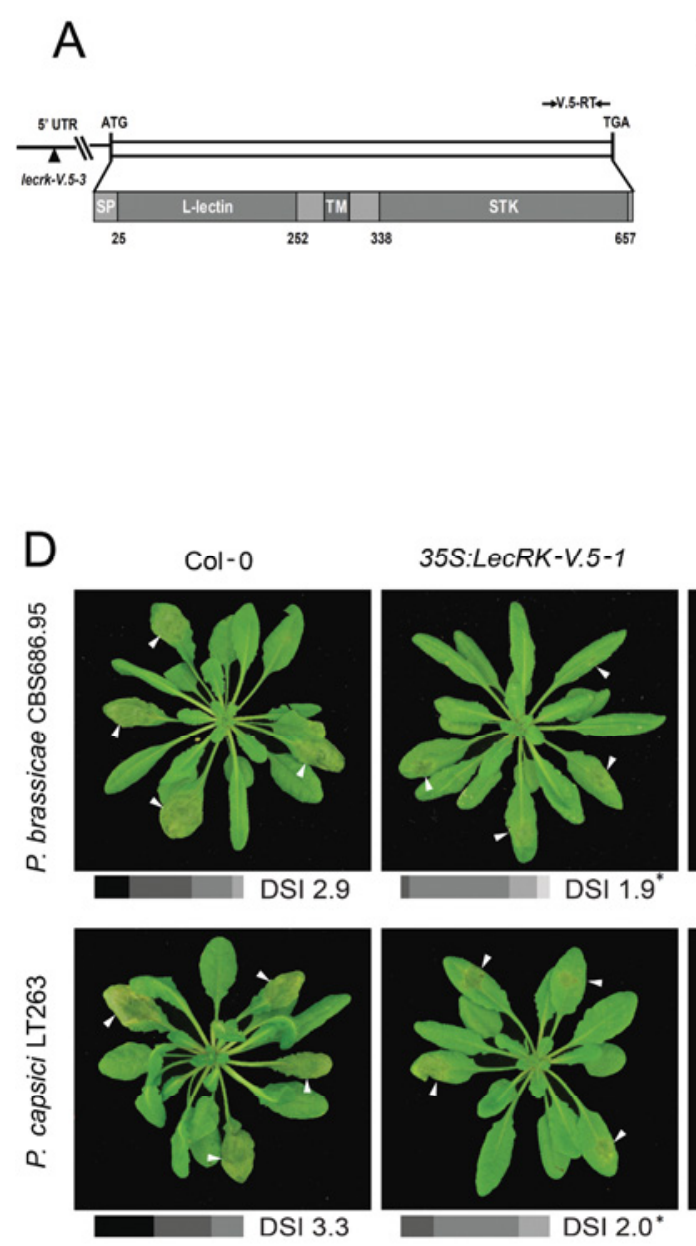

$\mathrm{F}$

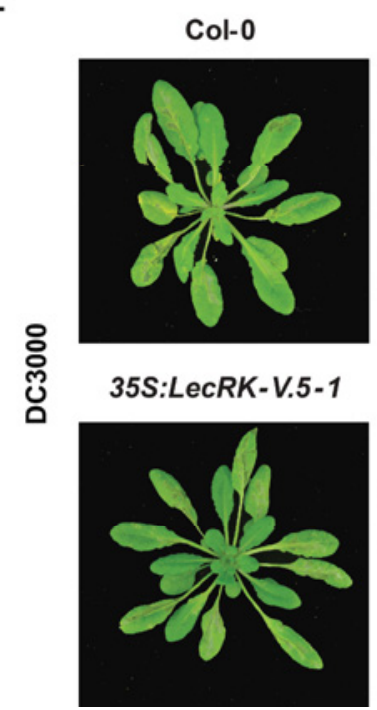

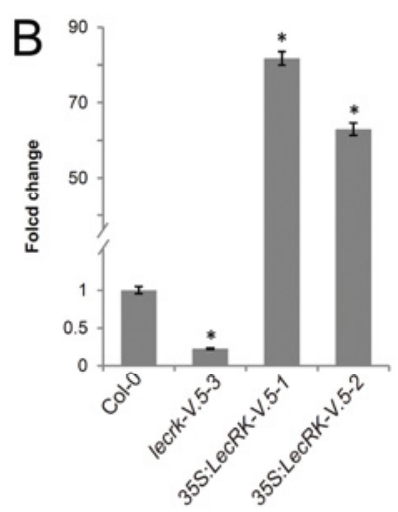

35S:LecRK-V.5-2
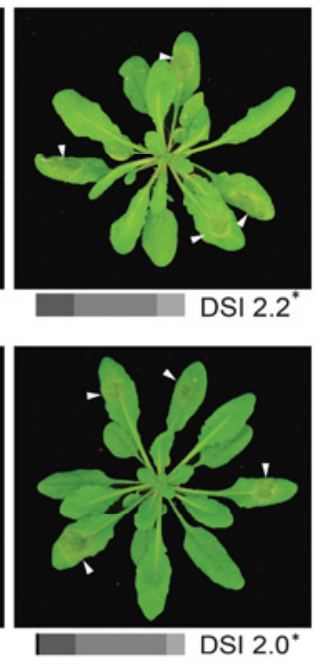
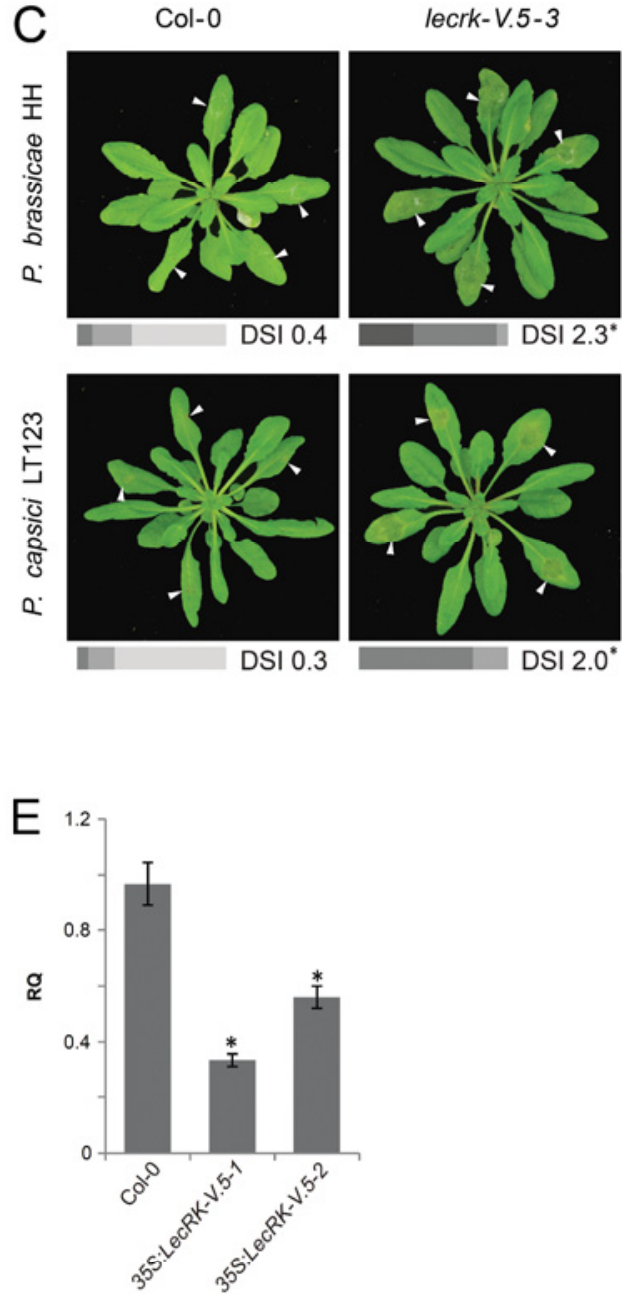

Fig. 3. LecRK-V.5 plays opposite roles in resistance to Phytophthora spp. and Pseudomonas syringae. A, Schematic model representing LecRK-V.5. The black arrowhead points to the T-DNA insertion site in lecrk-V.5-3 while the head-to-head arrow pairs indicate the position of the primers (V.5-RT) used for quantitative reverse-transcriptase polymerase chain reaction (qRT-PCR). B, qRT-PCR analysis of LecRK-V.5 transcript levels in Col-0, lecrk-V.5-3, and two overexpression lines; * indicates a significant difference $(P<0.05)$ according to a $t$ test. C, Disease symptoms on Col-0 and lecrk-V.5-3 4 days after inoculation (dpi) with Phytophthora brassicae HH and P. capsici LT123. White arrowheads point to inoculated leaves. Each bar represents the disease severity index (DSI) obtained from at least 30 leaves in three independent experiments, with the number showing the average DSI and an asterisk $(*)$ indicating a significant difference $(P<0.05)$ according to a $t$ test. D, Disease symptoms on Col- 0 and two LecRK-V.5 overexpression lines 3 dpi with $P$. brassicae CBS686.95 and P. capsici LT263. E, Relative quantification of $P$. capsici biomass (RQ) in Col-0 and overexpression lines at 3 dpi by qRT-PCR. Bars represent mean values ( \pm standard deviation $[\mathrm{SD}]$ ) relative to that of infected Col- 0 leaves; $*$ indicates significantly less biomass $(P<0.05)$, according to a $t$ test. Experiments were repeated twice with similar results. F, Disease symptoms on Col-0, lecrk-V.5-3, and two overexpression lines 4 dpi with Pseudomonas syringae DC3000. G, Bacterial growth in Col-0, lecrk-V.5-3, and two overexpression lines at 3 dpi. Bars shown are mean values ( \pm SD); * indicates significantly lower or higher bacterial titers $(P<0.05)$ according to a $t$ test. Experiments were repeated twice with similar results. 
with similar expression patterns were grouped together using hierarchical clustering with Pearson's correlation coefficient $(r)$ and average linkage. Pearson's $r$ is a metric to evaluate the strength of the correlation between variables, ranging from -1 to 1 (Usadel et al. 2009); therefore, we used the cut-off value of $r \geq 0.6$ for clustering which, in general, indicates strong positive correlation (Aoki et al. 2007; Fukushima et al. 2012). With this criterion, LecRK could be grouped into five clusters based on their expression patterns upon infection with $P$. infestans (Fig. 4C) and another five clusters upon infection with DC3000 (Fig. 4D). Of the 14 LecRK of which T-DNA insertion lines showed altered susceptibility to Phytophthora spp., five were grouped in cluster II and showed a slight reduction in expression between 6 and $24 \mathrm{hpi}$. The four LecRK that grouped in cluster $\mathrm{V}$ showed an opposite expression pattern compared with $L e c R K$ in cluster II. Of the remaining $L e c R K$, two are in cluster I (i.e., LecRK-I.9 and LecRK-S.1), two in cluster III (i.e., LecRK-S.4 and LecRK-S.7), and one in cluster IV (i.e., LecRK-III.2). Similar transcriptome-to-phenotype patterns were observed upon challenging with DC3000 (Fig. 4D). Of the five LecRK of which T-DNA insertion lines showed altered susceptibility to DC3000, LecRK-V.5 and LecRK-VI.2 are combined in cluster I, LecRK-S.1 and LecRK-S.4 are in cluster II, while LecRK-IV.4 was allocated to cluster III. When combining the expression patterns of the two time courses obtained from leaves inoculated with the two different pathogens, 27 of the 45 LecRK grouped in clusters, 11 in total, while the remaining 18 did not share their expression pattern with any other LecRK (Fig. 4B). Although the four LecRK that are implicated in resistance to both Phytophthora pathogens and DC3000 do not group in one cluster and are far apart in the dendrogram, the clustering of two and three relevant $L e c R K$ is maintained (i.e., LecRK-V.4 and LecRK-VIII. 2 and the combination of LecRK-V.5, LecRK-VI.2, and LecRK-IX.2).

\section{DISCUSSION}

Most of the plant immune receptors that have been identified to date are either plasma membrane-localized receptors, including RLK and receptor-like proteins (RLP, or intracellular nucleotide-binding leucine-rich repeat proteins (NLR) (Spoel and Dong 2012). Most were discovered based on their ability to activate defense responses upon recognition of MAMPs or pathogen effectors. For example, the $R L K$ gene encoding the MAMP receptor FLS2 was isolated by a forward genetics approach based on screening random mutants for flagellin-insensitive mutants, followed by map-based cloning of the locus harboring the mutation (Gómez-Gómez and Boller 2000). Also, the isolation of several NLR genes that function as major resistance genes in gene-for-gene interactions was the result of combining phenotype (i.e., pathogen recognition) and mapbased cloning; in this case, facilitated by linkage mapping using segregating progenies of resistant and susceptible parents (Marone et al. 2013; Rodewald and Trognitz 2013). Genome sequencing has revealed that defense-related RLK and NLR often have many homologs of which the function is unknown. They are grouped into families and subfamilies based on sequence similarity and shared domain architecture.

In this study, we focused on one such RLK subfamily in Arabidopsis, namely, the LecRK family, of which three of 45 members were previously identified as critical players in Arabidopsis innate immunity. Two of the three were selected for functional studies based on their increased expression in plants treated with priming agent BABA (LecRK-VI.2) (Singh et al. 2012) or oligogalacturonic acid (LecRK-V.5) (DesclosTheveniau et al. 2012). The third (LecRK-I.9) was selected via a phage display aimed at identifying RGD-binding pro- teins in plants (Gouget et al. 2006) and appeared to be a potential host target of a $P$. infestans RXLR effector (LecRK-I.9) (Bouwmeester et al. 2011). We anticipated that functional screening of other members of the LecRK family would reveal several novel candidates with a role in disease resistance. In an unbiased strategy, we monitored all available LecRK T-DNA insertion lines and, indeed, in addition to LecRK-I.9, LecRKV.5, and LecRK-VI.2 mentioned above, we found 16 additional LecRK of which mutants showed phenotypic changes in response to pathogens. T-DNA insertion lines are effective tools for determining gene function and linking genotype to phenotype (Lloyd and Meinke 2012; O'Malley and Ecker 2010). In other studies, similar unbiased screenings of T-DNA insertion line collections resulted in novel insight into gene function. An example is the study of ten Hove and associates (2011), who performed large-scale screening of T-DNA insertion lines of root-expressed leucine-rich repeat RLK and identified RLK implicated in hormone and abiotic stress responses. In a similar way, Wang and associates (2008) performed a screening of a broad range of T-DNA insertion lines in 56 genes encoding RLP and assigned functions to these RLP in plant development and resistance. It should be noted that the success rate in these two studies was relatively low; only a few T-DNA insertion lines were found that could be linked to the phenotype of interest. This could well be due to redundancy in function among members of these subfamilies. When one gene is inactivated because of the T-DNA insertion, another family member might take over its function, resulting in the wild-type phenotype. For example, SOMATIC EMBRYOGENESIS RECEPTOR KINASE 1 (SERK1) and the closest homolog SERK2 were concluded to function redundantly in controlling anther development and male gametophyte maturation based on the observation that the single mutants serk1 and serk 2 did not show any developmental defects, whereas serk1serk2 double mutants were defective in pollen development (Colcombet et al. 2005). This implies that such screenings, including ours, are by definition not conclusive for all members of the family; the ones of which mutants don't show a phenotype could still have an important role. Another issue to keep in mind is the variation in knock-out efficiency among mutants with a T-DNA insertion in the same gene. According to a survey on 1,084 published Arabidopsis insertion lines, an insertion in the coding region leads to inactivation of the target gene in over $98 \%$ of the cases; in mutants with an insertion before the start codon or after the stop codon, this percentage decreases but is still high (86 and 75\%, respectively) (Wang 2008). Variation in knock-out efficiency might explain why, in some cases, we found differences in phenotype among T-DNA insertion lines of the same gene such as, for example, the three LecRK-I.3 lines. In one case, we analyzed expression of the target gene (i.e., LecRK-IV.2), and we, indeed, detected residual expression in a mutant that contains a T-DNA insertion in the $5^{\prime}$ UTR, and no change in phenotype, whereas a line with insertions in the exon of that same gene showed a phenotype (Wan et al. 2008). In the case of $L e c R K-V .2$, we even found that a TDNA insertion in the $3^{\prime}$ UTR led to increased transcript levels of the target gene, a phenomenon that was also noted in the survey of Wang (2008). Thus, it is not surprising that we could not confirm the phenotype of a LecRK-V.2 mutant observed by Deng and associates (2009). Because we have not systematically checked all T-DNA insertion lines for expression, there is a chance that some of them are not true mutants, in the sense that they lost gene function. This implies that LecRK with TDNA insertion lines showing no phenotype might still be relevant for pathogen resistance.

In the unbiased strategy that we used here, successful identification of mutants is highly dependent on the design and 
A

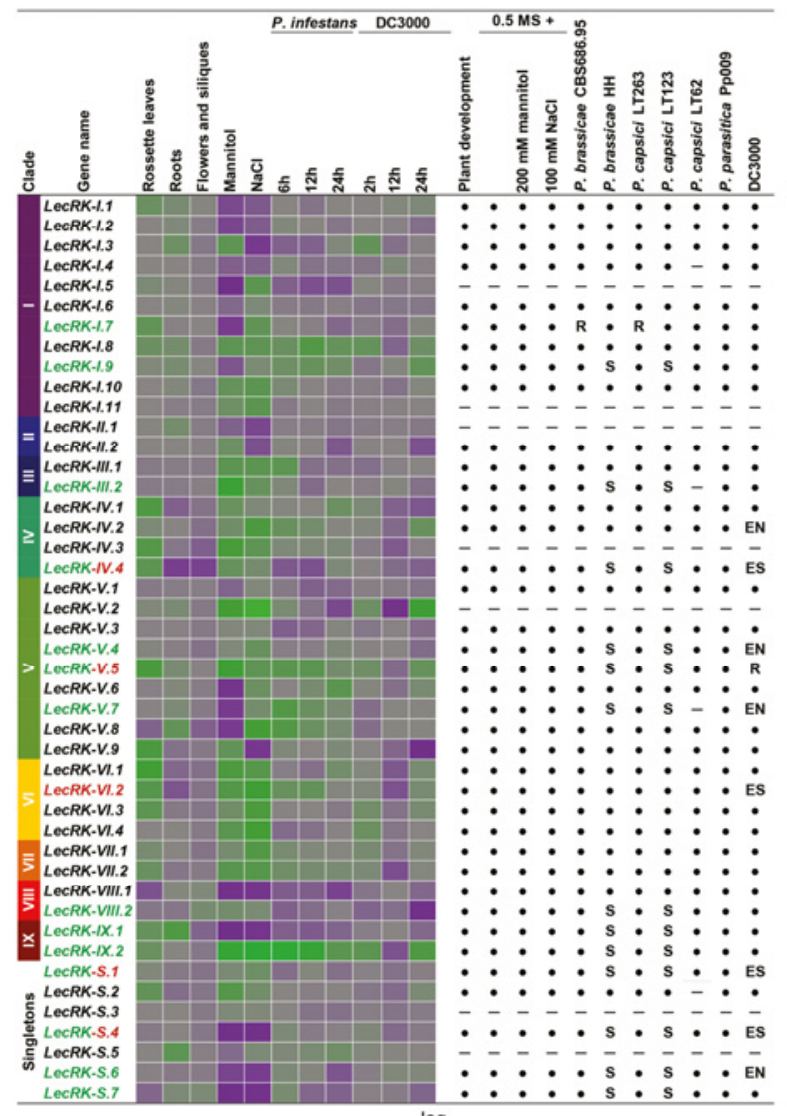

$\log _{2}$

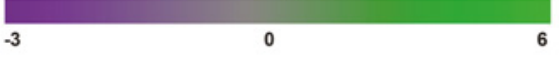

C

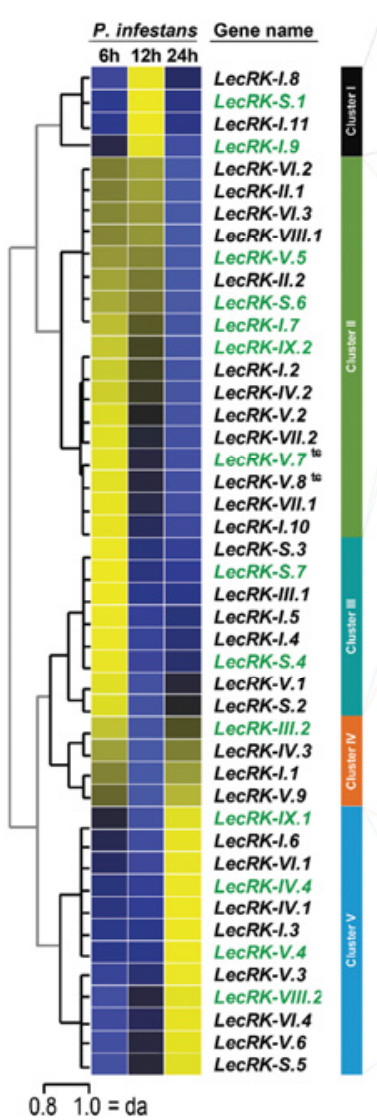

Expression patterns
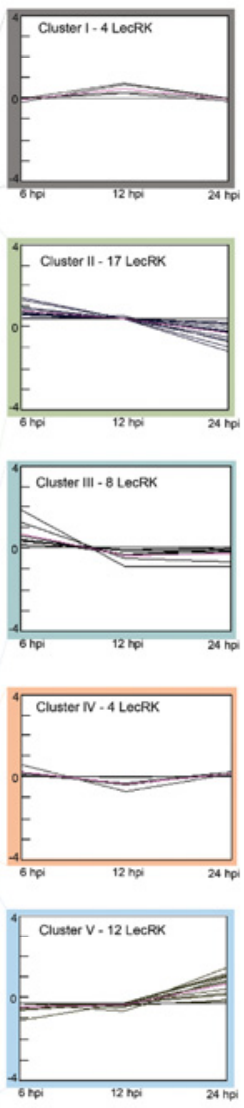

B

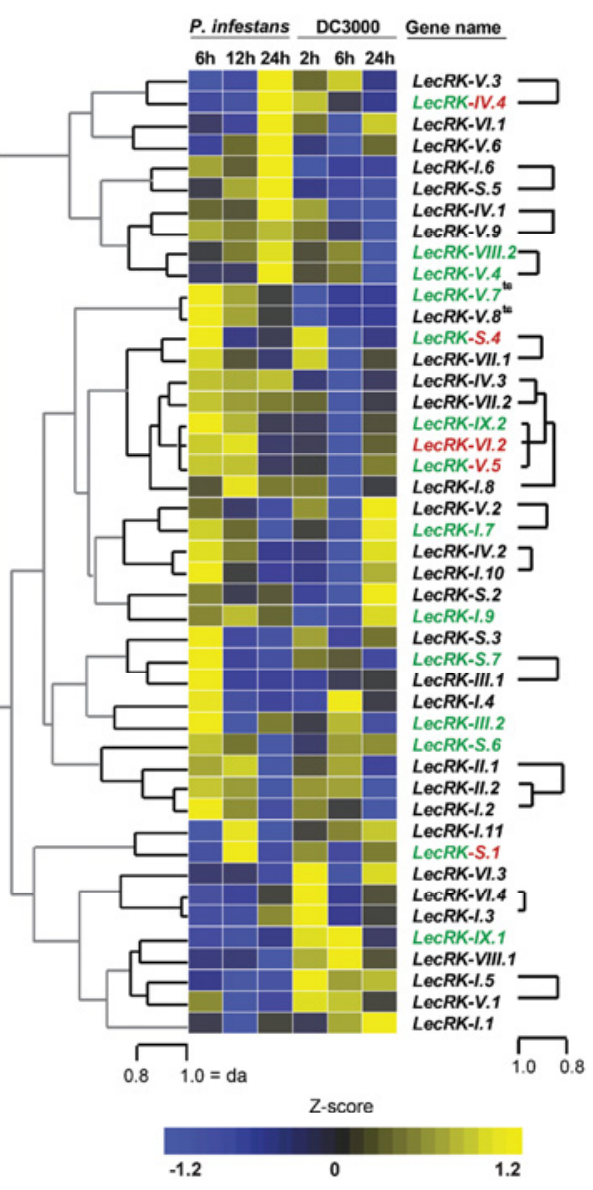

D Expression patterns
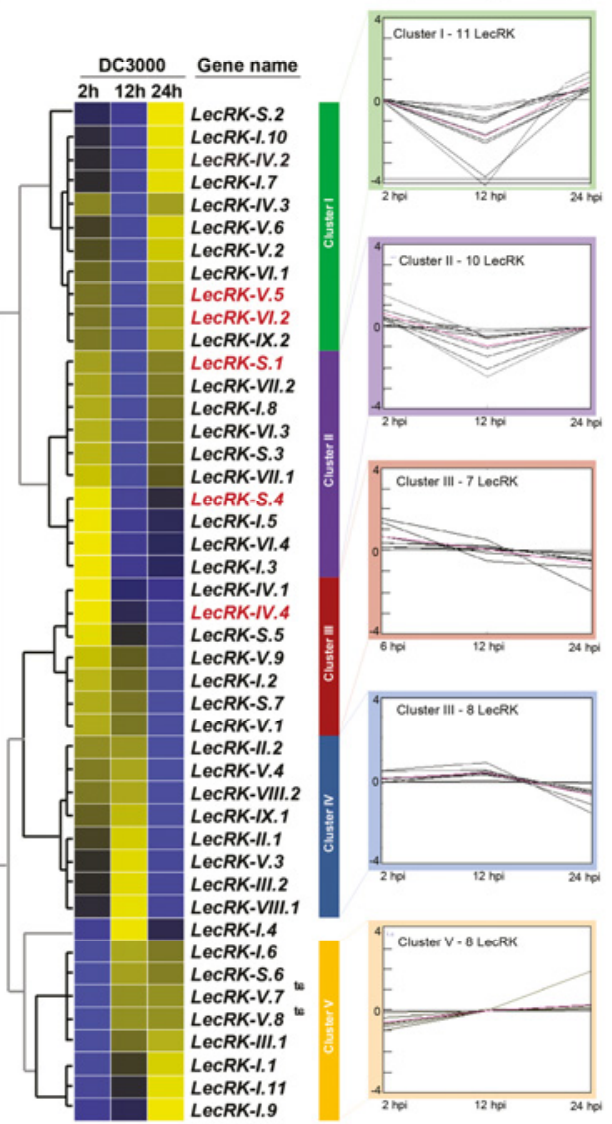

$0.81 .0=\mathrm{da}$ 
methodology of the phenotypic screening. In our screening, none of the LecRK T-DNA insertion lines showed phenotypic changes upon exposure to mannitol or $\mathrm{NaCl}$, despite the fact that multiple $L e c R K$ are induced during abiotic stress. It could well be that the conditions that we used for screening were not optimal. We only tested one concentration of mannitol and one of $\mathrm{NaCl}$ instead of a whole range of concentrations. When screening for disease resistance, the choice of pathogen isolates is extremely important. In the large-scale screening performed by Wang and associates (2008), none of the four RLP30 T-DNA insertion lines showed altered susceptibility toward the necrotrophic fungal pathogens Sclerotinia sclerotiorum and $\mathrm{B}$. $\mathrm{Ci}$ nerea. Recently, however, it was found that two of these $R L P 30$ T-DNA insertion lines showed increased susceptibility to $S$. sclerotiorum and $B$. cinerea, which is likely due to the fact that Zhang and associates (2013) used other isolates that are more virulent. In our screening, we choose to use different Phytophthora spp. and different isolates next to each other. The P. capsici and P. brassicae isolates were selected based on their differential behavior on Arabidopsis (Roetschi et al. 2001; Wang et al. 2013). We confirmed the role of LecRK-I.9 in resistance to $P$. brassicae and showed that this LecRK also inhibits $P$. capsici. In addition, we found several T-DNA insertion lines representing $12 \mathrm{LecRK}$ with the same phenotype as lecrk-I.9-1 and lecrk-I.9-2 (i.e., compromised resistance to both Phytophthora spp.). For four of these LecRK (i.e., LecRK-IV.4, LecRK-VIII.2, LecRK-IX.1, and LecRK-S.4), two or more independent T-DNA insertion lines showed consistent gain of susceptibility, demonstrating that they function as Phytophthora resistance components. For another one (i.e., LecRK-V.5), we confirmed the mutant phenotype by showing that overexpression of LecRK-V.5 enhances Phytophthora resistance. For the remaining ones, we have to be more cautious; in the phenotypic analysis, only a single T-DNA insertion line was included (the only one available) or, in the case of LecRK-S.7, only one of the two lines was a reliable mutant. Therefore, for these LecRK, only a potential role in resistance against Phytophthora pathogens can be suggested. With respect to $P$. brassicae and $P$. capsici, LecRK T-DNA insertion lines showed no specificity, suggesting that LecRK do not discriminate between Phytophthora spp. Overall, the T-DNA insertion lines are more susceptible to $P$. brassicae $\mathrm{HH}$ than to $P$. capsici LT123 and less susceptible to P. brassicae CBS686.95 than to $P$. capsici LT263. None of the LecRK T-DNA insertion lines was infected by the less virulent isolates $P$. capsici LT62 and $P$. parasitica $\mathrm{Pp} 009$. These two isolates are probably too weak to reach the stage at which LecRK come into play a as barrier for infection.

Four of the LecRK that have a function in Phytophthora resistance were also found to have a role in the interaction of Arabidopsis with the bacterial pathogen DC3000. Three of these promote resistance, suggesting that they can monitor dif- ferent types of pathogens and potentially confer a broad-spectrum resistance. The fourth one, however, LecRK-V.5, was found to have opposite roles in these two pathosystems (i.e., a resistance component against Phytophthora pathogens but a susceptibility factor for DC3000). One explanation for this dual role is that Phytophthora spp. and bacteria have different modes to enter host tissues. Bacteria usually penetrate via natural opening such as stomata, and stomatal closure can essentially restrict bacterial invasion (Melotto et al. 2006). Phytophthora pathogens can also penetrate via stomata but, instead, they prefer to penetrate at the junctions between epidermal cells (Hardham 2007; Wang et al. 2011). Therefore, the function of LecRK-V.5 in suppressing stomatal immunity is dispensable for preventing Phytophthora penetration. In addition, LecRK-V.5 was found negatively regulating the ABA response in Arabidopsis (Desclos-Theveniau et al. 2012). ABA plays a multifaceted role in regulating plant resistance (Ton et al. 2009). On the one hand, ABA enhances resistance against bacterial pathogens by regulating stomatal closure; on the other hand, ABA compromises defense to various pathogens via suppression of salicylic acid-signaling (Cao et al. 2011; Mohr and Cahill 2007; Sanchez-Vallet et al. 2012), a process that in Arabidopsis is essential for resistance to $P$. capsici (Wang et al. 2013). Hence, the gain of susceptibility found in lecrk-V.5-3 upon Phytophthora infection might be linked to ABA signaling.

Previously, LecRK-VI.2 was found to be required for resistance to various bacterial pathogens but not to the fungal pathogen $B$. cinerea (Huang et al. 2014; Singh et al. 2013). In our study, we confirmed the role of LecRK-VI.2 in bacterial resistance and found that it is not required for resistance to either Phytophthora pathogens or the fungal pathogen A. brassicicola. This specificity could be explained by the recent finding that LecRK-VI. 2 associates with FLS2 and participates in flg22mediated defense (Huang et al. 2014), which is essential for bacterial pathogen resistance but might be dispensable for resistance to Phytophthora or fungal pathogens.

Considering the fact that the primary sequence of a protein is a prime determinant of biological function, LecRK with high sequence similarity might share similar biological function. This might be the case for the four LecRK in clade I of the T-DNA insertion lines that showed compromised resistance to A. brassicicola. This clustering was not observed for LecRK involved in Phytophthora or DC3000 resistance. They are dispersed over different clades and, thus, sequence similarity as such does not account for a similar function. Moreover, the computed expression profiling revealed that LecRK with similar function displayed rather different expression patterns upon pathogen infection and, therefore, expression is also not a determinant of the functional similarity among $L e c R K$.

In summary, phenotypic analysis of a large set of T-DNA insertion lines uncovered multiple Arabidopsis LecRK with

Fig. 4. Compilation of expression profiles of $L e c R K$ and phenotypes of LecRK T-DNA insertion lines. A, The heat map in the left panel shows $L e c R K$ expression levels in different plant tissues and in response to various biotic and abiotic stresses. Signal intensity $\log _{2}$ ratios are color-coded in the range of -3 (purple) to 6 (green), as indicated in the scale bar, and represent the relative expression levels compared with corresponding controls. The table on the right shows the phenotypes of the LecRK T-DNA insertion lines, with ' $\mathrm{R}$ ' standing for resistant, 'S' for susceptible, 'EN' for enhanced necrotic symptoms but no increased bacterial proliferation, and 'ES' for enhanced necrotic symptoms and increased bacterial proliferation; a black dot $(\bullet)$ indicates no consistent phenotypic changes and - indicates not tested. B to D, Hierarchical clustering of LecRK based on expression patterns at $\mathbf{C}, 6,12$, and $24 \mathrm{~h}$ after inoculation with Phytophthora infestans; D, 2, 6, and $24 \mathrm{~h}$ after inoculation with Pseudomonas syringae DC3000; or B, combined using Pearson's correlation coefficient $(r)$ of per gene Z-score transformed $\log _{2}$ values (sample relative to control). Clusters were created using a cut-off value of $r \geq 0.6$. C, and $\mathbf{D}$, Clusters are indicated by vertical colored bars connected to panels showing LecRK expression patterns in each cluster. The lengths of the branches (da) are the scaled (black lines) and unscaled (gray lines) average Pearson correlation distances $(\mathrm{dp}=2 \mathrm{da}=1-r)$. Normalized expression data are color coded to indicate higher (yellow, saturated at 1.2) and lower (blue, saturated at -1.2) expression relative to the respective controls. On the microarray, the same probes represent LecRK-V.7 and LecRK-V.8 and, thus, the expression values are identical. A to D, LecRK involved in Phytophthora resistance (S or R) are depicted in green and those involved in DC3000 resistance (ES or R) in dark red. LecRK involved in both are depicted in combined green-red. 
thus-far-unknown function that contribute to defense against pathogens, thus pointing to LecRK as potential immune receptors. The incentive for this screening was the finding that LecRK-I.9 is a Phytophthora resistance component and, as importantly, that transgenic potato lines expressing Arabidopsis LecRK-I. 9 are more tolerant to P. infestans, the causal agent of potato late blight (Bouwmeester et al. 2014). This suggests that LecRK can be exploited to confer resistance in crops. Because LecRK are widespread in the plant kingdom, understanding the function of LecRK in Arabidopsis will facilitate the functional analysis of LecRK in crop species, and this will be helpful in exploring novel resistance sources against damaging plant pathogens. Further studies should reveal how the different LecRK are activated, what their ligands are, and how they feed into downstream signaling and activate defense. The overall importance of LecRK in plants is demonstrated by the recent finding that LecRK-I.9 (alias DORN1) is capable of binding extracellular ATP (Choi et al. 2014). It is the first eATP receptor identified in plants and has a completely different structure than eATP receptors in animals. The collection of LecRK T-DNA insertion lines described in this study will be instrumental to further explore the role of LecRK as eATP receptors.

\section{MATERIALS AND METHODS}

\section{Analysis of T-DNA insertion lines.}

LecRK T-DNA insertion lines were obtained from the European Arabidopsis Stock Center (Alonso et al. 2003). T-DNA insertion sites and homozygosity were checked by PCR using T-DNA primers LBb1.3 (SALK lines), LB1 (SAIL lines), or p745 (WiscDsLox lines) in combination with gene-specific primers.

\section{Plant growth conditions.}

Arabidopsis seed were sown on soil or in vitro on $0.5 \mathrm{MS}$ medium, stratified in the dark at $4{ }^{\circ} \mathrm{C}$ for 3 days, and subsequently placed in a growth chamber at 19 to $21^{\circ} \mathrm{C}$ with 75 to $78 \%$ relative humidity and a 12 -h photoperiod. Alternatively, seed were grown on vertically orientated $0.5 \mathrm{MS}$ plates or 0.5 MS plates supplemented with $100 \mathrm{mM} \mathrm{NaCl}$ or $200 \mathrm{mM}$ mannitol or under the same conditions as described previously (Deng et al. 2009). Seedling growth was evaluated from the 7 th to 16th day after seed sowing. Each treatment included at least three replicates and the experiment was repeated four times.

\section{Pathogen growth and infection assays.}

$P$. brassicae, $P$. capsici, and $P$. parasitica were grown in the dark on $20 \%$ (vol/vol) V8 agar plates at appropriate temperatures. Infection assays on Arabidopsis with fresh mycelial plugs were carried out as described previously (Bouwmeester et al. 2011). Disease symptom scoring using a DSI and the Phytophthora biomass quantification were conducted as described by Wang and associates (2013).

Pseudomonas syringae DC3000 was grown on King's B medium supplemented with rifampicin at $50 \mu \mathrm{g} \mathrm{ml}^{-1}$. Arabidopsis plants were spray inoculated with bacterial suspensions with a concentration of $10^{8} \mathrm{CFU} \mathrm{ml}^{-1}$ in $10 \mathrm{mM} \mathrm{MgSO}_{4}$ and $0.02 \%$ (vol/vol) Silwet-L77, or mock treated with $10 \mathrm{mM}$ $\mathrm{MgSO}_{4}$ and $0.02 \%$ (vol/vol) Silwet-L77. Bacterial growth was determined at $3 \mathrm{dpi}$. Whole rosettes were collected and measured for fresh weight and subsequently homogenized in 10 $\mathrm{mM} \mathrm{MgSO}_{4}$. Homogenates were serially diluted in $10 \mathrm{mM}$ $\mathrm{MgSO}_{4}$ and were plated on Pseudomonas agar (BD Biosciences) containing rifampicin at $50 \mu \mathrm{g} \mathrm{ml}^{-1}$. Bacterial growth was evaluated by counting bacterial CFU 2 days after incuba- tion at $28^{\circ} \mathrm{C}$ and normalized as CFU per gram using the total weight of the inoculated rosette leaves. This experiment was repeated three times, each with at least three replicates.

A. brassicicola MUCL20297 was cultured in darkness at $22^{\circ} \mathrm{C}$ on potato dextrose agar. Four- to five-week-old Arabidopsis rosette leaves were drop inoculated with $5 \mu$ of spore suspensions $\left(10^{6}\right.$ spores $\left.\mathrm{ml}^{-1}\right)$ or mock inoculated with $5 \mu \mathrm{l}$ of water. Plants were placed in trays covered with lids and kept in the dark during the first $24 \mathrm{~h}$. Thereafter, they were moved to a climate chamber with the aforementioned settings. Disease symptoms were checked from 3 to $5 \mathrm{dpi}$.

\section{Plasmid construction and plant transformation.}

The coding sequence of LecRK-V.5 was amplified by PCR using Pfu DNA polymerase (Promega) with primers listed in Supplementary Table S2. Purified PCR products were cloned into the vector $\mathrm{pENTR/D-TOPO} \mathrm{(Invitrogen).} \mathrm{Plasmids} \mathrm{with}$ the correct sequence were recombined by LR reaction into the binary vector $\mathrm{pGWB} 2$. The resulting vector was transformed into Agrobacterium tumefaciens GV3101. Arabidopsis Col-0 plants were transformed using the floral dip method, as previously described (Zhang et al. 2006).

\section{RNA isolation and qRT-PCR.}

Total RNA was isolated from 4-week-old Arabidopsis leaves with a NucleoSpin RNA plant kit (Macherey-Nagel) and thereafter used as the template for cDNA synthesis with an oligodT primer using an M-MLV reverse transcriptase kit (Promega). qRT-PCR was performed using an ABI7300 real-time PCR system (Applied Biosystems). Data were analyzed using a $2^{-\Delta \Delta C T}$ method (Livak and Schmittgen 2001). Transcript levels of LecRK were normalized with Arabidopsis Actin2 and expressed as mean fold changes ( \pm standard deviation) relative to that in Col-0 leaves, which was arbitrarily set as 1 .

\section{Expression data analysis.}

LecRK expression data during plant growth or upon treatment with different stimuli were obtained from the public microarray datasets using eFP-Browser at the Bio-Array Resource (Winter et al. 2007). Signal intensity $\log _{2}$ ratios of the samples relative to respective controls were used as a measure for LecRK expression. Of the datasets sampled from Arabidopsis in different growth stages and exposed to abiotic stresses, the maximal (highest or lowest) signal intensity relative to the mean throughout all arrays in the database was retrieved. Expression data of Arabidopsis treated with biotic stresses were derived from 5-week-old Arabidopsis Col-0 rosette leaves inoculated with Phytophthora infestans or infiltrated with DC3000. LecRK-V.7 (At3g59740) and LecRK-V.8 (At3g59750) share the same probe-set and obtained the same values. For expression pattern analysis, per-gene $\log _{2}$ values were normalized by the Z-score method according to Cheadle and associates (2003). Coexpression analysis of LecRK was conducted using hierarchical clustering with Pearson's correlation coefficient $(r)$ and average linkage (da), as implemented in MultiExperiment Viewer (MeV4.0).

\section{ACKNOWLEDGMENTS}

We thank L. Zimmerli (National Taiwan University) for providing LecRK-VI.2 mutants, M. F. Seidl (Wageningen University) for discussion, and K. Oluwaseun (University of Ibadan) for technical assistance. This project was financially supported by a Wageningen University sandwich Ph.D. fellowship (Y. Wang), a Huygens scholarship (Y Wang), and a VENI grant (K. Bouwmeester) from the Netherlands Organization for Scientific Research (Technology Foundation NWO-STW) and the Foodfor-Thought-Thought-for-Food campaign of the Wageningen University Fund. 


\section{LITERATURE CITED}

Alonso, J. M., Stepanova, A. N., Leisse, T. J., Kim, C. J., Chen, H. M., Shinn, P., Stevenson, D. K., Zimmerman, J., Barajas, P., Cheuk, R., Gadrinab, C., Heller, C., Jeske, A., Koesema, E., Meyers, C. C., Parker, H., Prednis, L., Ansari, Y., Choy, N., Deen, H., Geralt, M., Hazari, N., Hom, E., Karnes, M., Mulholland, C., Ndubaku, R., Schmidt, I., Guzman, P., Aguilar-Henonin, L., Schmid, M., Weigel, D., Carter, D. E., Marchand, T., Risseeuw, E., Brogden, D., Zeko, A., Crosby, W. L., Berry, C. C., and Ecker, J. R. 2003. Genome-wide insertional mutagenesis of Arabidopsis thaliana. Science 301:653-657.

Aoki, K., Ogata, Y., and Shibata, D. 2007. Approaches for extracting practical information from gene co-expression networks in plant biology. Plant Cell Physiol. 48:381-390.

Boller, T., and Felix, G. 2009. A renaissance of elicitors: Perception of microbe-associated molecular patterns and danger signals by patternrecognition receptors. Annu. Rev. Plant Biol. 60:379-407.

Bouwmeester, K., and Govers, F. 2009. Arabidopsis L-type lectin receptor kinases: Phylogeny, classification, and expression profiles. J. Exp. Bot. 60:4383-4396.

Bouwmeester, K., de Sain, M., Weide, R., Gouget, A., Klamer, S., Canut, H., and Govers, F. 2011. The lectin receptor kinase LecRK-I.9 is a novel Phytophthora resistance component and a potential host target for a RXLR effector. PLoS Pathog. 7:e1001327.

Bouwmeester, K., Han, M., Blanco-Portales, R., Song, W., Weide, R., Guo, L. Y., van der Vossen, E. A. G., and Govers, F. 2014. The Arabidopsis lectin receptor kinase LecRK-I.9 enhances resistance to Phytophthora infestans in Solanaceous plants. Plant Biotechnol. J. 12:1016.

Cao, F. Y., Yoshioka, K., and Desveaux, D. 2011. The roles of ABA in plant-pathogen interactions. J. Plant Res. 124:489-499.

Cheadle, C., Vawter, M. P., Freed, W. J., and Becker, K. G. 2003. Analysis of microarray data using $\mathrm{Z}$ score transformation. J. Mol. Diagn. 5:7381.

Chinchilla, D., Shan, L., He, P., de Vries, S., and Kemmerling, B. 2009. One for all: The receptor-associated kinase BAK1. Trends Plant Sci. 14:535-541

Choi, J., Tanaka, K., Cao, Y., Qi, Y., Qiu, J., Liang, Y., Lee, S. Y., and Stacey, G. 2014. Identification of a plant receptor for extracellular ATP. Science 343:290-294.

Colcombet, J., Boisson-Dernier, A., Ros-Palau, R., Vera, C. E., and Schroeder, J. I. 2005. Arabidopsis SOMATIC EMBRYOGENESIS RECEPTOR KINASES1 and 2 are essential for tapetum development and microspore maturation. Plant Cell 17:3350-3361.

Deng, K., Wang, Q., Zeng, J., Guo, X., Zhao, X., Tang, D., and Liu, X. 2009. A lectin receptor kinase positively regulates ABA response during seed germination and is involved in salt and osmotic stress response. J. Plant Biol. 52:493-500.

Desclos-Theveniau, M., Arnaud, D., Huang, T. Y., Lin, G. J. C., Chen, W. Y., Lin, Y. C., and Zimmerli, L. 2012. The Arabidopsis lectin receptor kinase LecRK-V.5 represses stomatal immunity induced by Pseudomonas syringae pv. tomato DC3000. PLoS Pathog. 8:e1002513.

Dievart, A., Dalal, M., Tax, F. E., Lacey, A. D., Huttly, A., Li, J. M., and Clark, S. E. 2003. CLAVATA1 dominant-negative alleles reveal functional overlap between multiple receptor kinases that regulate meristem and organ development. Plant Cell 15:1198-1211.

Eisen, M. B., Spellman, P. T., Brown, P. O., and Botstein, D. 1998. Cluster analysis and display of genome-wide expression patterns. Proc. Natl. Acad. Sci. U.S.A. 95:14863-14868.

Fukushima, A., Nishizawa, T., Hayakumo, M., Hikosaka, S., Saito, K., Goto, E., and Kusano, M. 2012. Exploring tomato gene functions based on coexpression modules using graph clustering and differential coexpression approaches. Plant Physiol. 158:1487-1502.

Gómez-Gómez, L., and Boller, T. 2000. FLS2: An LRR receptor-like kinase involved in the perception of the bacterial elicitor flagellin in Arabidopsis. Mol. Cell 5:1003-1011.

Gouget, A., Senchou, V., Govers, F., Sanson, A., Barre, A., Rouge, P., Pont-Lezica, R. P., and Canut, H. 2006. Lectin receptor kinases participate in protein-protein interactions to mediate plasma membrane-cell wall adhesions in Arabidopsis. Plant Physiol. 140:81-90.

Hardham, A. R. 2007. Cell biology of plant-oomycete interactions. Cell. Microbiol. 9:31-39.

Hervé, C., Dabos, P., Galaud, J. P., Rougé, P., and Lescure, B. 1996. Characterization of an Arabidopsis thaliana gene that defines a new class of putative plant receptor kinases with an extracellular lectin-like domain. J. Mol. Biol. 258:778-788.

Huang, P., Ju, H. W., Min, J. H., Zhang, X., Kim, S. H., Yang, K. Y., and Kim, C. S. 2013. Overexpression of L-type lectin-like protein kinase 1 confers pathogen resistance and regulates salinity response in Arabidopsis thaliana. Plant Sci. 203:98-106.
Huang, P. Y., Yeh, Y. H., Liu, A. C., Chien, C. P., and Zimmerli, L. 2014 The Arabidopsis LecRK-VI.2 associates with the pattern-recognition receptor FLS2 and primes Nicotiana benthamiana pattern-triggered immunity. Plant J. 79:243-255.

Huitema, E., Vleeshouwers, V. G. A. A., Francis, D. M., and Kamoun, S. 2003. Active defence responses associated with non-host resistance of Arabidopsis thaliana to the oomycete pathogen Phytophthora infestans. Mol. Plant Pathol. 4:487-500.

Li, X. L., Tan, Y. C., and Ng, S. K. 2006. Systematic gene function prediction from gene expression data by using a fuzzy nearest-cluster method. BMC Bioinf. 7:S23.

Liebrand, T. W. H., van den Burg, H. A., and Joosten, M. H. A. J. 2013. Two for all: Receptor-associated kinases SOBIR1 and BAK1. Trends Plant Sci. 19:123-132.

Livak, K. J., and Schmittgen, T. D. 2001. Analysis of relative gene expression data using real-time quantitative PCR and the $2^{-\Delta \Delta C T}$ method. Methods 25:402-408.

Lloyd, J., and Meinke, D. 2012. A comprehensive dataset of genes with a loss-of-function mutant phenotype in Arabidopsis. Plant Physiol. 158:1115-1129.

Marone, D., Russo, M. A., Laido, G., De Leonardis, A. M., and Mastrangelo, A. M. 2013. Plant nucleotide binding site-leucine-rich repeat (NBSLRR) genes: Active guardians in host defense responses. Int. J. Mol. Sci. 14:7302-7326.

Melotto, M., Underwood, W., Koczan, J., Nomura, K., and He, S. Y. 2006. Plant stomata function in innate immunity against bacterial invasion. Cell 126:969-980.

Mohr, P., and Cahill, D. 2007. Suppression by ABA of salicylic acid and lignin accumulation and the expression of multiple genes, in Arabidopsis infected with Pseudomonas syringae pv. tomato. Funct. Integr. Genomics 7:181-191.

O'Malley, R. C., and Ecker, J. R. 2010. Linking genotype to phenotype using the Arabidopsis unimutant collection. Plant J. 61:928-940.

Rodewald, J., and Trognitz, B. 2013. Solanum resistance genes against Phytophthora infestans and their corresponding avirulence genes. Mol. Plant Pathol. 14:740-757.

Roetschi, A., Si-Ammour, A., Belbahri, L., Mauch, F., and Mauch-Mani, B. 2001. Characterization of an Arabidopsis-Phytophthora pathosystem: Resistance requires a functional $P A D 2$ gene and is independent of salicylic acid, ethylene and jasmonic acid signalling. Plant J. 28:293305.

Sanchez-Vallet, A., Lopez, G., Ramos, B., Delgado-Cerezo, M., Riviere, M. P., Llorente, F., Fernandez, P.V., Miedes, E., Estevez, J. M., Grant, M., and Molina, A. 2012. Disruption of abscisic acid signaling constitutively activates Arabidopsis resistance to the necrotrophic fungus Plectosphaerella cucumerina. Plant Physiol. 160:2109-2124.

Shiu, S. H., and Bleecker, A. B. 2003. Expansion of the receptor-like kinase/Pelle gene family and receptor-like proteins in Arabidopsis. Plant Physiol. 132:530-543.

Singh, P., Kuo, Y. C., Mishra, S., Tsai, C. H., Chien, C. C., Chen, C. W., Desclos-Theveniau, M., Chu, P. W., Schulze, B., Chinchilla, D., Boller, T., and Zimmerli, L. 2012. The lectin receptor kinase-VI.2 is required for priming and positively regulates Arabidopsis pattern-triggered immunity. Plant Cell 24:1256-1270.

Singh, P., Chien, C. C., Mishra, S., Tsai, C. H., and Zimmerli, L. 2013. The Arabidopsis LECTIN RECEPTOR KINASE-VI.2 is a functional protein kinase and is dispensable for basal resistance to Botrytis cinerea. Plant Signal. Behav. 8:1-3.

Spoel, S. H., and Dong, X. 2012. How do plants achieve immunity? Defence without specialized immune cells. Nat. Rev. Immunol.12:89-100.

ten Hove, C. A., Bochdanovits, Z., Jansweijer, V. M. A., Koning, F. G., Berke, L., Sanchez-Perez, G. F., Scheres, B., and Heidstra, R. 2011. Probing the roles of LRR-RLK genes in Arabidopsis thaliana roots using a custom T-DNA insertion set. Plant Mol. Biol. 76:69-83.

Ton, J., Flors, V., and Mauch-Mani, B. 2009. The multifaceted role of ABA in disease resistance. Trends Plant Sci. 14:310-317.

Usadel, B., Obayashi, T., Mutwil, M., Giorgi, F. M., Bassel, G. W., Tanimoto, M., Chow, A., Steinhauser, D., Persson, S., and Provart, N. J. 2009. Co-expression tools for plant biology: Opportunities for hypothesis generation and caveats. Plant Cell Environ. 32:1633-1651.

van Wees, S. C. M., Chang, H. S., Zhu, T., and Glazebrook, J. 2003. Characterization of the early response of Arabidopsis to Alternaria brassicicola infection using expression profiling. Plant Physiol. 132:606617.

Wan, J., Patel, A., Mathieu, M., Kim, S. Y., Xu, D., and Stacey, G. 2008. A lectin receptor-like kinase is required for pollen development in Arabidopsis. Plant Mol. Biol. 67:469-482.

Wang, G., Ellendorff, U., Kemp, B., Mansfield, J. W., Forsyth, A., Mitchell, K., Bastas, K., Liu, C. M., Woods-Toer, A., Zipfel, C., de Wit, P. J. G. M., Jones, J. D. G., Toer, M., and Thomma, B. P. H. J. 2008. A genome- 
wide functional investigation into the roles of receptor-like proteins in Arabidopsis. Plant Physiol. 147:503-517.

Wang, Y. 2008. How effective is T-DNA insertional mutagenesis in Arabidopsis? J. Biochem. Technol. 1:11-20.

Wang, Y., Meng, Y., Zhang, M., Tong, X., Wang, Q., Sun, Y., Quan, J., Govers, F., and Shan, W. 2011. Infection of Arabidopsis thaliana by Phytophthora parasitica and identification of variation in host specificity. Mol. Plant Pathol. 12:187-201.

Wang, Y., Bouwmeester, K., Van de Mortel, J. E., Shan, W., and Govers, F. 2013. A novel Arabidopsis-oomycete pathosystem: Differential interactions with Phytophthora capsici reveal a role for camalexin, indole glucosinolates and salicylic acid in defence. Plant Cell Environ. 36:11921203.

Winter, D., Vinegar, B., Nahal, H., Ammar, R., Wilson, G. V., and Provart, N. J. 2007. An "Electronic Fluorescent Pictograph" browser for exploring and analyzing large-scale biological data sets. PLoS One 2:e718.

Xin, Z., Wang, A., Yang, G., Gao, P., and Zheng, Z. L. 2009. The Arabi- dopsis A4 subfamily of lectin receptor kinases negatively regulates abscisic acid response in seed germination. Plant Physiol. 149:434-444.

Zhang, W., Fraiture, M., Kolb, D., Löffelhardt, B., Desaki, Y., Boutrot, F. F. G., Tör, M., Zipfel, C., Gust, A. A., and Brunner, F. 2013. Arabidopsis RECEPTOR-LIKE PROTEIN30 and receptor-like kinase SUPPRESSOR OF BIR1-1/EVERSHED mediate innate immunity to necrotrophic fungi. Plant Cell 25:4227-4241.

Zhang, X., Henriques, R., Lin, S. S., Niu, Q. W., and Chua, N. H. 2006. Agrobacterium-mediated transformation of Arabidopsis thaliana using the floral dip method. Nat. Protocols 1:641-646.

\section{AUTHOR-RECOMMENDED INTERNET RESOURCES}

SALK Institute Genome Analysis Laboratory: signal.salk.edu European Arabidopsis Stock Center: Arabidopsis.info Bio-Array Resource: www.bar.utoronto.ca 